\title{
Constraint-induced breaking and restoration of ergodicity in spin-1 PXP models
}

\author{
Bhaskar Mukherjee $\odot,{ }^{1,2,{ }^{*}}$ Zi Cai, ${ }^{1,3,4, \dagger}$ and W. Vincent Liu $\odot^{2,1,5,4,6, *}$ \\ ${ }^{1}$ Wilczek Quantum Center, School of Physics and Astronomy, Shanghai Jiao Tong University, Shanghai 200240, China \\ ${ }^{2}$ Department of Physics and Astronomy, University of Pittsburgh, Pittsburgh, Pennsylvania 15260, USA \\ ${ }^{3}$ Key Laboratory of Artificial Structures and Quantum Control, School of Physics and Astronomy, \\ Shanghai Jiao Tong University, Shanghai 200240, China \\ ${ }^{4}$ Shanghai Research Center for Quantum Sciences, Shanghai 201315, China \\ ${ }^{5}$ T. D. Lee Institute, Shanghai Jiao Tong University, Shanghai 200240, China \\ ${ }^{6}$ Department of Physics and Shenzhen Institute for Quantum Science and Engineering, \\ Southern University of Science and Technology, Shenzhen 518055, China
}

(Received 23 April 2021; revised 10 August 2021; accepted 11 August 2021; published 31 August 2021)

\begin{abstract}
Eigenstate thermalization hypothesis (ETH) has played a pivotal role in understanding ergodicity and its breaking in isolated quantum many-body systems. The recent experiment on 51-atom Rydberg quantum simulator by Bernien et al. [Nature (London) 551, 579 (2017)] and subsequent theoretical analysis have shown that hardcore kinetic constraint can lead to weak ergodicity breaking. In this work, we demonstrate, using $1 d$ spin-1 PXP models, that miscellaneous type of ergodicity can be realized by adjusting the hardcore constraints between different components of nearest-neighbor spins. This includes ETH violation due to emergent shattering of Hilbert space into exponentially many subsectors of various sizes, a novel form of nonintegrability with an extensive number of local conserved quantities and strong ergodicity. We analyze these different forms of ergodicity and study their impact on the nonequilibrium dynamics of a $\mathbb{Z}_{2}$ initial state. We use forward-scattering approximation to understand the amount of $\mathbb{Z}_{2}$ oscillation present in these models. Our work shows that not only ergodicity breaking but an appropriate choice of constraints can lead to restoration of ergodicity as well.
\end{abstract}

DOI: 10.1103/PhysRevResearch.3.033201

\section{INTRODUCTION}

Eigenstate thermalization hypothesis (ETH) offers the most widely accepted mechanism of thermalization of local observables in out-of-equilibrium closed quantum many-body systems [1-5]. An ETH satisfying system, prepared in an unentangled product state, gets strongly entangled quickly under its own dynamics, losing all the information of the initial state except the conserved quantities (e.g., total energy). These systems are usually strongly interacting in nature which makes the full quantum system, though well isolated from external environment, suffer from the presence of an indigenous heat bath. This makes the study of ETH-violating systems not only of fundamental importance but also of technological importance from the perspective of quantum information protection, quantum state preparation and preservation of quantum coherence (which is the measure of quantumness of a system) up to very long time.

\footnotetext{
*bhaskarmukherjee1991@gmail.com

'zcai@sjtu.edu.cn

${ }^{\ddagger}$ wvliu@ pitt.edu
}

Published by the American Physical Society under the terms of the Creative Commons Attribution 4.0 International license. Further distribution of this work must maintain attribution to the author(s) and the published article's title, journal citation, and DOI.
Integrable systems [6], possessing an extensive number of conserved quantities, have long been known to disobey ETH. A prototypical example is transverse field Ising model which though appears an interacting system in original spin language, becomes a free system via Jordan-Wigner transformation [7]. Many-body localized systems [8-12] which are mostly one-dimensional interacting quantum system with onsite disorder potential, forms another class of ETH violating system and has been studied in detail over the past decade. These systems are examples where we see strong violation of ETH in the sense all eigenstates violates ETH.

Recently, anomalous oscillation from a density wave $\left(\mathbb{Z}_{2}\right)$ state observed in a quench dynamics experiment using a 51-atom Rydberg quantum simulator [13] has revitalized the interest into the field of thermalization and its violation. This phenomenon is understood by using spin- $1 / 2$ PXP model which hosts extensive number of ETH violating states with high $\mathbb{Z}_{2}$ overlap, dubbed as quantum many-body scars, in its spectrum $[14,15]$. So far, a plethora of study [16-20] has not only revealed the full phenomenology of the scar states but they have been found in a variety of models ranging from different interacting spin chains [21-23], Hubbard models [24,25], higher spin PXP models [26,27], Floquet systems [28-33], disordered systems [34], quantum Hall system [35], in higher dimension [36-41], via confinement in Ising spin chains [42] as well as in lattice gauge theories [43-45]. In many of these studies scar states are exactly constructed either in matrix product state (MPS) form or by repeatedly acting 
suitably designed creation operator on some mother state. These kind of construction quite straightforwardly explains the ETH violating nature of the scar states. But in spite of being the first experimentally realized model to host quantum many-body scar, a fully satisfactory explanation of scarring in PXP model is still an open problem. Except a few scar states for which exact MPS representation was obtained [16], numerics and semianalytical techniques like forward-scattering approximation [14,15,19], single-mode approximation [46], etc., are the only option to study the majority number of scar states in the PXP model.

In this work we focus on spin-1 PXP model [26,27] which is defined by the Hamiltonian: $H=-\sum_{i} \mathcal{P} S_{i}^{x} \mathcal{P}$ in a chain of $L$ sites where the local (per site) Hilbert space is spanned by the eigenstates of $S^{z}(|m\rangle \equiv|-\rangle,|0\rangle,|+\rangle$ for $m=-1,0,+1)$ and the operator $\mathcal{P}=\prod_{i} \mathcal{P}_{i, i+1}$ characterizes the constrained Hilbert space. In traditional spin-1 PXP [26] model at least one of two consecutive spin must be in the $|-\rangle$ state which fixes the form of the projector: $\mathcal{P}_{i, i+1}=P_{i}+P_{i+1}-P_{i} P_{i+1}$ with $P_{i}=|-\rangle_{i}\left\langle-\left.\right|_{i}\right.$. This means, $\left.\mid 00\right\rangle,|+0\rangle,|0+\rangle$, and $|++\rangle$ type of configurations are not allowed in the constrained Hilbert space $\left(\mathbb{H}^{\mathrm{PXP}}\right)$. This opens up the question that what happen when different set of constraints are used. In this work we show that the many-body spectrum of spin-1 PXP model can get dramatically changed when certain constraints are abolished. To this end we consider three different set of constraints and construct three corresponding model Hamiltonians. The plan of the paper is as follows: In Sec. II we introduce the models and define their Hamiltonians. We also present the main ergodic features of the three models in this section. In Sec. III we explore the ergodicity properties of different models by studying dynamics starting from a $\mathbb{Z}_{2}$ initial state which we further understand by using forward-scattering approximation. The fate of ergodic properties of all the three models under different perturbations is studied in Sec. IV. Finally, we conclude and discuss future prospects in Sec. V.

\section{MODELS AND RESULTS}

Models I, II, and III are defined by the following Hamiltonians:

$$
H^{\alpha}=\sum_{i=1}^{L} \mathcal{P}^{\alpha} S_{i}^{x} \mathcal{P}^{\alpha},
$$

where $\mathcal{P}^{\alpha}=\prod_{i} \mathcal{P}_{i, i+1}^{\alpha}$ for $\alpha=I, I I, I I I$ and $\mathcal{P}_{i, i+1}^{\mathrm{I}}=\mathcal{P}_{i, i+1}+$ $\left[(|+\rangle\langle+|)_{i} \otimes(|+\rangle\langle+|)_{i+1}\right], \quad \mathcal{P}_{i, i+1}^{\mathrm{II}}=\mathbb{I}_{i} \otimes \mathbb{I}_{i+1}-\left[(|0\rangle\langle 0|)_{i} \otimes\right.$ $\left.(|0\rangle\langle 0|)_{i+1}\right], \quad \mathcal{P}_{i, i+1}^{\mathrm{II}}=\mathcal{P}_{i, i+1}^{\mathrm{II}}-\left[(|+\rangle\langle+|)_{i} \otimes(|+\rangle\langle+|)_{i+1}\right]$. Note that, these three models are also in PXP form, but to distinguish them from traditional spin-1 PXP model we use the model index $(\alpha)$ in the superscript.

In Fig. 1 we show how these models can be obtained by imposing specific constraints over a spin-1 free paramagnet $\left(H=\sum_{i} S_{i}^{x}\right)$ or abolishing specific constraints from traditional spin-1 PXP model. We find that if we allow $|++\rangle$ configurations on top of $\mathbb{H}^{\mathrm{PXP}}$, which we call model $\mathrm{I}$, then the spectrum gets shattered into exponentially many emergent subsectors of different size including $\mathbb{H}^{\mathrm{PXP}}$ as one of the largest block. If we further allow $|+0\rangle /|0+\rangle$ type configurations (model II), then an extensive number of local conserved

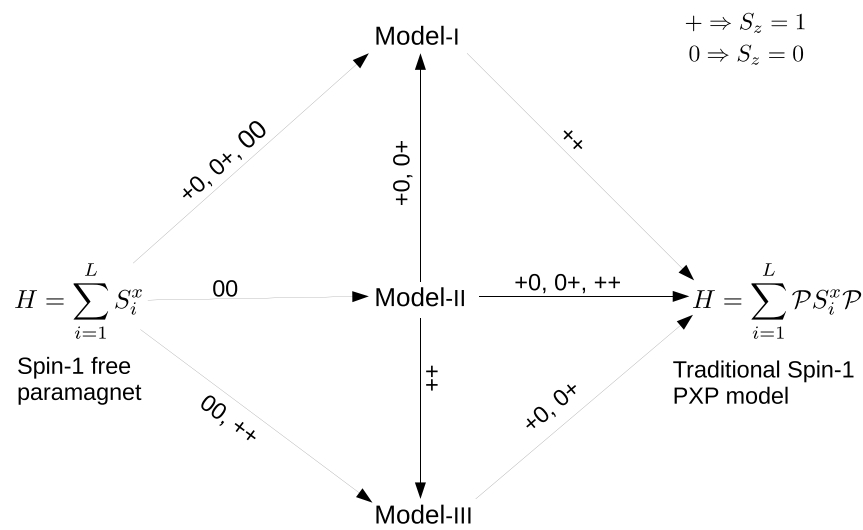

FIG. 1. Schematic picture, showing the construction of models I, II, and III by imposing (along the arrow direction) constraints on the Hilbert space of a spin-1 free paramagnet and abolishing (opposite to the arrow direction) constraints from the traditional spin-1 PXP model. The constraints (forbidden configurations on a pair of adjacent sites) are shown on top of the arrows.

quantities arises. This does not make model II exactly solvable due to some degeneracies in the spectrum of the conserved quantities. In fact, we find that these conserved quantities can be used at most to label different sectors of model II which are nothing but disconnected patches of spin-1/2 PXP model of different sizes. If we disallow $|++\rangle$ type configurations on top of model II, then all the scar states disappear and the spectrum become strongly ergodic (model III).

The symmetries of these Hamiltonians include translation, inversion about center bond/site and particle-hole symmetry characterized by the vanishing anticommutator of the operator $\mathcal{C}=\prod_{i}\left[2\left(S_{i}^{z}\right)^{2}-I_{i}\right]$ with the Hamiltonians: $\left\{H^{\alpha}, \mathcal{C}\right\}=0$ for $\alpha=I, I I, I I I$. The last symmetry guarantees that if there is an eigenstate $\psi$ at energy $E$, then there will also be an eigenstate $(\mathcal{C}|\psi\rangle)$ at $-E$. The Hilbert space dimension grows much slowly than naive $3^{L}$ depending on the nature of constraints and choice of boundary conditions (see Table I). We utilize the first two symmetry and work in zero momentum and inversion symmetric $(K=0, I=+1)$ sector to access largest possible system. The intertwining of the particle-hole and inversion symmetry generates an exponentially large number of zero modes $[15,47,48]$.

The spectrum of model I posses a lot of degeneracies at nonzero (including integers). We explore the connectivity of states in the Hilbert space of model I and find that it (even each momentum and inversion symmetry sector) is shattered into exponentially many emergent subsectors $[49,50]$ [see Fig. 2(a)]. The lowest possible size (in $S^{z}$ basis) of such emergent blocks is one, hence these are unentangled, zero energy eigenstates of $H^{\mathrm{I}}$. These inert states remain frozen under the dynamics generated by the Hamiltonian. The number of such inert eigenstates $\left(I_{L}\right)$ scales as $I_{L} \sim \phi^{L}$ in large $L$ limit where $\phi=\frac{\sqrt{5}-1}{2}$ is the golden ratio and the proportionality constant depends on the choice of boundary condition (see Appendix B). The eigenstates in low $(>1)$ dimensional subsectors have very small but nonzero entanglement, some of them also have interesting properties like integer energy and magnetization. The minimally entangled 
TABLE I. Hardcore constraints, scaling of Hilbert space dimension in OBC (see Appendix A for derivations) and main features of the spectrum of models I, II, and III.

\begin{tabular}{lccc}
\hline \hline & Model I & Model II & Model III \\
\hline Forbidden configurations & $|00\rangle,|+0\rangle,|0+\rangle$ & $|00\rangle$ & $|00\rangle,|++\rangle$ \\
$d_{L}^{\text {OBC }}$ & $\approx 2.247^{L}$ & $\frac{(1-\sqrt{3})^{L}(\sqrt{3}-2)+(1+\sqrt{3})^{L}(\sqrt{3}+2)}{2 \sqrt{3}} \approx 2.732^{L}$ & $\frac{(1-\sqrt{2})^{L+1}+(1+\sqrt{2})^{L+1}}{2} \approx 2.414^{L}$ \\
Feature & Emergent Hilbert & Extensive number of local conserved & quantities; nonintegrable \\
& space shattering & qugly ergodic & \\
\hline \hline
\end{tabular}

states (in the central region of the spectrum) are eigenstates of a subsector of size $3 \times 3$. These eigenstates (with energy $E= \pm 1,0)$ are given by $\left|\psi_{E}\right\rangle=\frac{1}{2}\left|\psi_{-}\right\rangle-\frac{e^{i \pi E}}{2}\left|\psi_{+}\right\rangle+\frac{E}{\sqrt{2}}\left|\psi_{0}\right\rangle$ where $\left|\psi_{m}\right\rangle=\frac{1}{\sqrt{L}} \sum_{n=1}^{L} T^{n}\left[\otimes_{i=1}^{L-3}|+\rangle_{i} \otimes|-\mathbf{m}-\rangle\right] ; \mathbf{m}= \pm, 0$ and $T$ is the translation operator. Red (blue bold) color is used to denote inert (active) sites. The half chain entanglement entropy $\left(S_{L / 2}\right)$ of these states are found to be $S_{L / 2}=$ $\ln \left(\frac{2 L}{L-4}\right)+\frac{4}{L} \ln \left(\frac{L-4}{2}\right)$ (see Appendix B) which assumes area law behavior in thermodynamic limit [see inset of Fig. 2(b)]. We also find that some special states with $E= \pm 2$ (belonging to subsectors of size $6 \times 6$ ) has logarithmic entanglement entropy $\left(S_{L / 2}=\ln L-\ln 2\right.$; see Appendix B). The magnetization $\left(S_{z}=\sum_{i=1}^{L} S_{i}^{z}\right)$ of such eigenstates with energy $E= \pm n$ turns out to be $L-5 n$ which are non-negative at any system size as these type of states do not appear for $L<5 n$ (see Appendix B). This expectation value is much higher than the corresponding thermal average $\left(\left\langle S_{z}\right\rangle_{\beta}=\operatorname{Tr}\left[\rho_{\beta} S_{z}\right]\right.$ with $\rho_{\beta}=$ $\left.\exp \left(-\beta H^{I}\right) / \operatorname{Tr}\left[\exp \left(-\beta H^{I}\right)\right]\right)$ which is always negative due to the excessiveness of $|-\rangle$ compared to $|+\rangle$ states in the constrained Hilbert space of model I. Thus such states clearly violates ETH.

The number of $|++\rangle$ configurations $\left(N_{++}=\right.$ $\left.\sum_{i}(|++\rangle\langle++|)_{i, i+1}\right)$ turns out to be a conserved quantity for model I which can be used to label (not uniquely) different emergent subsectors. The block with $N_{++}=0$ is nothing but the traditional spin-1 PXP model. Note that, this kinetic constraint (no two spins in $|+\rangle$ state can sit next to each other) is emergent in nature as the underlying Hamiltonian $\left(H^{\mathrm{I}}\right)$ does not have it [51]. The block with $N_{++}=1$ is the largest block in the largest possible system size $(L=16)$ we have explored numerically. Blocks with same $N_{++}(>1)$ can be further labeled by $N_{+++}$and so on. We note that there are many subsectors with $N_{++}>1$ and $N_{+++}=0$ whose states contains isolated $|++\rangle$ configurations, separated by active sites. Such subsectors can be further labeled by number of $|++-++\rangle,|++--++\rangle$ etc type of inert configurations. For example, at $L=10$, there are three subsectors with $\left(N_{++}=2, N_{+++}=0\right)$ which can be uniquely labeled by the quantum numbers $\left(N_{++-++}, N_{++--++}\right)$with values $(0,0),(0,1)$ and $(1,0)$. For $L>10$, number of such
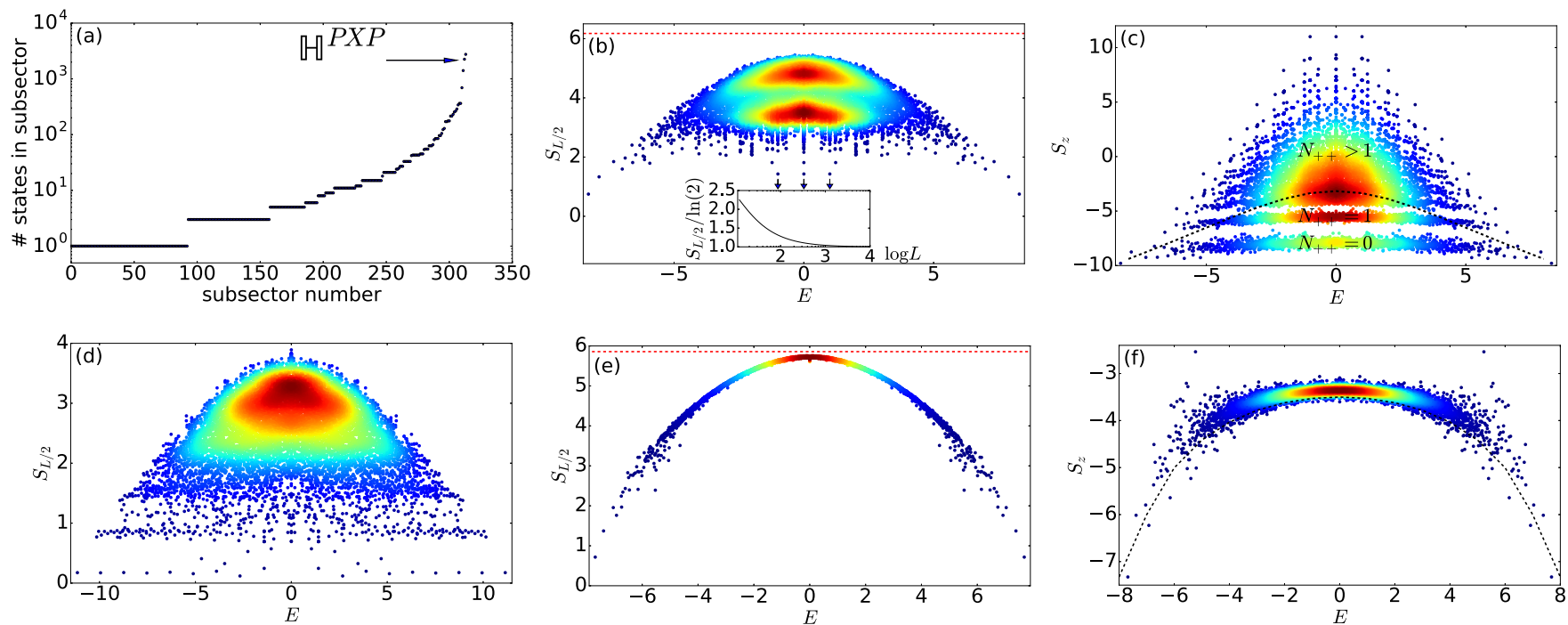

FIG. 2. Ergodicity property of models I, II, and III. (a) Shattering of Hilbert space in model I. Size of different emergent subsectors in $K=0, I=+1$ sector for $L=16$. The traditional spin-1 PXP model is the second largest sector at this system size. (b) $S_{L / 2}$ of all the eigenstates in $K=0, I=+1$ sector of model I for $L=16$. System size scaling of $S_{L / 2}$ for the three lowest entangled state is shown in the inset. (c) $S_{z}$ of all eigenstates in $K=0, I=+1$ sector of model I for $L=16$. The three cluster of states is characterized by the value of $N_{++}$. (d) $S_{L / 2}$ of all the eigenstates of model II which belong to the sectors where central $n(n=2, \ldots, L-2)$ sites are labeled by $O=+1$ and the rest are $O=-1, L=20$. (e) $S_{L / 2}$ and (f) $S_{z}$ of all the eigenstates in $K=0, I=+1$ sector of model III, $L=14$. In panels (b) and (e) the Page value of $S_{L / 2}$ is shown in red dashed line. For panel (d) the Page value $\left(S_{L / 2}^{\text {Page }}=9.625\right)$ is not shown. In panels (c) and (f) the canonical (Gibbs) ensemble prediction of $S_{z}$ is shown in black dashed line. In all the color plots, the regions with warmer colors have higher density of states. 


\section{$\mathrm{S}=1 / 2 \mathrm{PXP}$ Non-interacting $\mathrm{S}=1 / 2 \mathrm{PXP}$ Symmetry Sector $\ldots 0^{+} 0^{+} O^{+} O^{+} O^{-} O^{-} O^{-} O^{-} \quad O^{+} O^{+} O^{+} O^{+} \ldots$ Projection of $\left|\mathbb{Z}_{2}\right\rangle|\cdots \downarrow \downarrow \downarrow \downarrow| \otimes\left|0^{-} 0^{-} 0^{-} 0^{-}\right| \otimes|\downarrow \downarrow \downarrow \downarrow \cdots\rangle$ $\begin{array}{lll}\text { Rapid } & \text { Undamped } & \text { Rapid } \\ \text { thermalization } & \text { oscillations } & \text { thermalization }\end{array}$}

FIG. 3. Schematic structure of a typical symmetry sector of model II and projection of $\mathbb{Z}_{2}$ state in that sector.

subsectors is $>3$, consequently unique labeling of them can not be achieved. In fact, projectors on inert configurations of all system sizes $\leqslant L$ are conserved quantities of a system of size $L$. Though the inert states are unentangled, the projectors on them are nonlocal in nature. Moreover, their number scales exponentially with system size and thus an unique set of conserved quantities to distinguish different subsectors is lacking. That's why this is an emergent shattering of Hilbert space induced by the choice of constraints. The ratio of the size of $\mathbb{H}^{\mathrm{PXP}}$ (the largest emergent subsector for $L<16$ ) and the largest symmetry sector goes as $\sim(2 / 2.247)^{L} \equiv e^{-\gamma L}$, where $\gamma \sim 0.116>0$. This suggests that this is a strong Hilbert space fragmentation. We note that ETH violation due to emergent fragmentation of Hilbert space was first observed in fractonic circuit with local conservation of charge and dipole moment [49] and in the corresponding Hamiltonian system [50]. Subsequent works showed that Hilbert space fragmentation can also happen from strict confinement [52] and via frustration [53].

In model II only $|00\rangle$ type configurations are disallowed. The spectrum of model II also holds nonzero-energy degeneracies inside the $K=0, I=+1$ sector. This time we find an extensive $(=L)$ number of local quantities $\left(O_{i}\right)$ which commutes with the Hamiltonian, i.e., $\left[O_{i}, H^{\mathrm{II}}\right]=0, \forall i$. We find that $O_{i}=(|+\rangle\langle-|+| 0\rangle\langle 0|+|-\rangle\langle+|)_{i}=2\left(S_{i}^{x}\right)^{2}-\mathbb{I}_{i}$ (see Appendix C). Interestingly, this does not make model II completely integrable as each $O_{i}$ have degenerate eigenstates. The eigenvalues of $O_{i}$ are $(+1,+1,-1)$ with corresponding eigenstates $\left|O_{1}^{+}\right\rangle=\frac{1}{\sqrt{2}}(|+\rangle+|-\rangle),\left|O_{2}^{+}\right\rangle=|0\rangle,\left|O^{-}\right\rangle=$ $\frac{1}{\sqrt{2}}(|+\rangle-|-\rangle)$. Therefore all eigenstates of $H^{\mathrm{II}}$ cannot be uniquely labeled by the eigenvalues of all $O_{i}$ 's as the number of such available quantum numbers $\left(2^{L}\right)$ is far less than the total number of eigenstates $\left(\approx 2.7^{L}\right)$. Thus the conserved quantities can only be used to block diagonalize the Hamiltonian. The largest block is characterized by $O_{i}=+1, \forall i$. It is easy to see that this sector is equivalent to the celebrated spin-1/2 PXP model with the identification: $|\downarrow\rangle=\left|O_{1}^{+}\right\rangle$and $|\uparrow\rangle=$ $\left|O_{2}^{+}\right\rangle$. Thus, in principle one can expect to see all phenomenon observed in spin-1/2 PXP model in this spin-1 model also. On the theoretical side, Lin-Motrunich [16] type exact eigenstates can also be constructed here, not only for the largest sector but also for all sectors where island of sites with $O_{i}=+1$ are separated by sites with $O_{i}=-1$, as these sectors are nothing but disconnected patches of spin-1/2 PXP model of different sizes. For example, 16 exact states can be constructed for the symmetry sector shown in Fig. 3 (with one $\left|O^{-}\right\rangle$at each end to ensure open boundary condition (OBC) for the two PXP patches) with the following form:

$$
\left|\Psi_{m, n, q, r}^{L}\right\rangle=\left|O_{1}^{-}\right\rangle \otimes\left|\Phi_{m, n}^{\frac{L-6}{2}}\right\rangle \otimes_{i=1}^{4}\left|O_{\frac{L-4}{2}+i}^{-}\right\rangle \otimes\left|\Phi_{q, r}^{\frac{L-6}{2}}\right\rangle \otimes\left|O_{L}^{-}\right\rangle,
$$

where $\Phi_{x, y}^{l}=\sum_{\sigma} v_{x}^{T} B^{\sigma_{1}} C^{\sigma_{2}} \cdots B^{\sigma_{l-1}} C^{\sigma_{l}} v_{y}\left|\sigma_{1} \cdots \sigma_{l}\right\rangle, \quad x, y \in$ $\{1,2\}, \sigma_{i} \in\{\downarrow, \uparrow\}, v_{1}=(1,1)^{T}, v_{2}=(1,-1)^{T}$ and

$$
\begin{aligned}
B^{\downarrow} & =\left(\begin{array}{lll}
1 & 0 & 0 \\
0 & 1 & 0
\end{array}\right), \quad B^{\uparrow}=\sqrt{2}\left(\begin{array}{ccc}
0 & 0 & 0 \\
1 & 0 & 1
\end{array}\right), \\
C^{\downarrow} & =\left(\begin{array}{cc}
0 & -1 \\
1 & 0 \\
0 & 0
\end{array}\right), \quad C^{\uparrow}=\sqrt{2}\left(\begin{array}{cc}
1 & 0 \\
0 & 0 \\
-1 & 0
\end{array}\right) .
\end{aligned}
$$

The energies of the states in Eq. (2) are given by $E=\sqrt{2}(n-$ $m+r-q$ ) where $m, n, q, r \in\{1,2\}$. The entanglement entropy of these states can be zero or nonzero depending on the bipartition scheme of the full system. The smallest sector is of size 1 characterized by $O_{i}=-1$ at all sites, hence $H^{\mathrm{II}}$ posses an unentangled, exact zero energy eigenstate: $\prod_{i}\left|O_{i}^{-}\right\rangle$. We note that sectors where at least two consecutive sites have $O_{i}=+1$ feels the hardcore interaction whereas sectors where each $O_{i}=+1$ site is isolated by at least one $O_{i}=-1$ from both side is basically noninteracting in nature [54]. The number of such noninteracting sectors scales as $\sim \phi^{L}$. Thus though many sectors of this model is nonintegrable in nature there exist exponentially many eigenstates which violates ETH.

Model III does not allow $|00\rangle$ and $|++\rangle$ type configurations. This model neither have any conserved quantity (other than translation, inversion and particle-hole) nor its spectrum have any emergent shattering. Due to the high connectivity in Hilbert space [55], this model displays strongly ergodic behavior [see Figs. 2(e) and 2(f)] in spite of being a constrained system. We draw Hilbert space connectivity graph for all three models in Fig. 4. Nodes of the graphs denote the Fock states; two nodes are connected if matrix element of the Hamiltonian is nonzero in between those two states. In the graph theory language, Hamiltonian is nothing but the adjacency matrix of the corresponding graph. One can see that models I and II have disconnected portions in the Hilbert space as a consequence of constraint induced emergent fragmentation and presence of extensive number of conserved quantities respectively. But, the graph of model III is fully connected and more complicated than the largest connected portions of model I and model II. We note that, when we disallow $|++\rangle$ type configurations from model II and obtain model III, all the conserved quantities of model II are lost. Consequently, fragmentation of Hilbert space caused by the conserved quantities are also abolished. But the resulting model III could have emergent fragmentation as in model I, instead it has a fully connected Hilbert space. Now, even with a fully connected Hilbert space, a model can host quantum scars and display nonergodicity as in original spin-1/2 or spin-1 PXP models. We find that the Hilbert space connectivity graph of model III has some additional features on top of the same for the original spin-1 PXP model (largest connected portion of model I), namely satellite clusters at each corner of the connectivity graph. Though this does not lead to a drastic increase of average connectitvity, the local increase of coordination number helps the system to become ergodic and delocalize quickly throughout the entire 
(a)

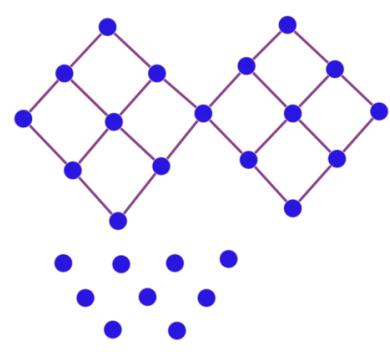

(b)

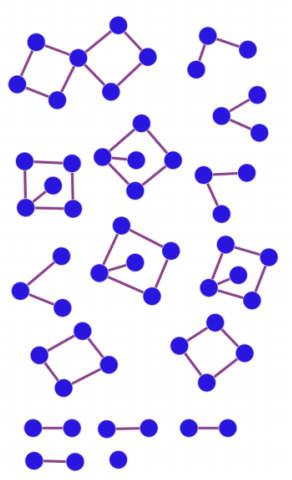

(c)

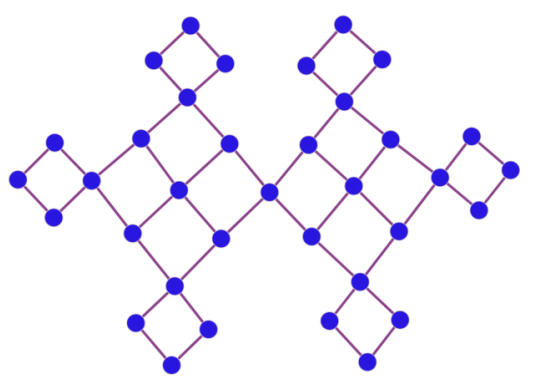

FIG. 4. Hilbert space connectivity graph of model I (a), model II (b), and model III (c) for $L=4$ (PBC). For models I and III, the nodes of the graphs denote the Fock states in $S^{z}$ basis where as for model II they denote product states in $O_{i}$ basis. There are 16 different clusters in (b) which corresponds to $2^{4}$ different symmetry sectors of model II for $L=4$. The largest cluster in (a) and (b) is equivalent to traditional spin-1 and spin-1/2 PXP model, respectively.

Hilbert space starting from any initial state. We consider this constraint-induced restoration of ergodicity in model III to be a new kind of geometric effect. In the next section we will analyze it more using forward-scattering approximation (FSA) and $\mathbb{Z}_{2}$ dynamics.

\section{III. $\mathbb{Z}_{2}$ DYNAMICS AND FSA}

The difference in ergodicity of models I, II, and III can be probed by studying the dynamics of local observables (we choose $\left.A=(|+\rangle\langle+|)_{i} \otimes(|+\rangle\langle+|)_{i+1}\right)$ from the initial state $\left|\mathbb{Z}_{2}\right\rangle=\otimes_{i=1}^{L / 2}|-\rangle_{2 i-1}|+\rangle_{2 i}$. It is worthy to point out here that all traditional spin-s PXP models studied so far exhibits long lived coherent oscillation starting from the $\mathbb{Z}_{2}$ state [26]. In our model I the $\mathbb{Z}_{2}$ state belong to the emergent subsector $\mathbb{H}^{\mathrm{PXP}}$ and so the corresponding dynamics will be exactly same as that of traditional spin-1 PXP model. On the other hand, $\left|\mathbb{Z}_{2}\right\rangle$ state has uniform overlap with all $2^{L}$ symmetry sectors of model II as can be seen from the expression: $\left|\mathbb{Z}_{2}\right\rangle=$ $2^{-L / 2}\left[\otimes_{i=1}^{L / 2}\left(\left|O_{1}^{+}\right\rangle-\left|O^{-}\right\rangle\right)_{2 i-1}\left(\left|O_{1}^{+}\right\rangle+\left|O^{-}\right\rangle\right)_{2 i}\right]$. Now how the state evolves inside a particular sector depends crucially on the interacting nature of that sector. The system will have perfect revival and no dephasing inside the fully noninteracting sectors, whereas inside the interacting sectors it will thermalize rapidly to infinite temperature (see Fig. 3). This is because the projection of the initial state in the interacting sectors corresponds to the fully polarized down state $(\cdots \downarrow \downarrow \downarrow \cdots)$, whereas the sectors themselves are nothing but disconnected patches of spin-1/2 PXP model of different sizes. So the $\mathbb{Z}_{2}$ dynamics in model II is a mixture of maximally thermal and maximally nonthermal effects, as a result neither it shows strong coherent oscillation nor thermalizes quickly [see Fig. 5(a)]. We note that though the number of fully noninteracting sectors $\left(\sim \phi^{L}\right)$ is a vanishingly small fraction $\left(\sim 0.81^{L}\right)$ of total number of sectors in thermodynamic limit, sectors with small interacting portions also increase exponentially with system sizes. Therefore the nature of the dynamics in thermodynamic limit is an interesting open question. Finally, we find that the $\mathbb{Z}_{2}$ dynamics in model III thermalizes rapidly to infinite temperature due to the strongly ergodic nature of the model. We show entanglement dynamics of the three model in Fig. 5(b). The initial growth of entanglement (which is related to the speed of information propagation) is fastest in model III, slowest in model I and intermediate in model II. This is consistent with the nature of the dynamics of local observable in these three models.

We now corroborate our dynamics results using FSA which has been very successful in capturing the scar states in large PXP systems $[14,15]$. We begin by decomposing $H^{\alpha}$ into two parts: $H^{\alpha}=H_{+}^{\alpha}+H_{-}^{\alpha}$ such that $H_{-}^{\alpha}$ annihilates the initial state $\left|v_{0}\right\rangle=\left|\mathbb{Z}_{2}\right\rangle$ and $H_{+}^{\alpha}=\left(H_{-}^{\alpha}\right)^{\dagger}$. The repeated application of $H_{+}^{\alpha}$ on $\left|\mathbb{Z}_{2}\right\rangle$ generates the FSA vectors $:\left|v_{n}\right\rangle=\frac{1}{\beta_{n}} H_{+}^{\alpha}\left|v_{n-1}\right\rangle$. For the spin-1 models in this paper, $n$ runs from 0 to $2 L$ after which the state gets annihilated. The FSA errors quantified by $\delta_{n}^{\alpha}=\| H_{-}^{\alpha}\left|v_{n}\right\rangle-\beta_{n}\left|v_{n-1}\right\rangle \|, n=1, \ldots, 2 L$ are a measure of damping force felt by the dynamical system due to many-body interaction effects. The system exhibits undamped oscillations when all the $\delta_{n}$ are zero which can be seen in a free paramagnet (no constraints) or by adding suitable perturbation of appropriate strength with the constrained model leading to the emergence of a near perfect SU(2) algebra [19]. Though, in general, FSA errors are nonzero in PXP type constrained systems considered here, it could be zero in first few steps depending on the nature of the constraints. For example, we find that in our model I, first four FSA steps are exact (i.e.,
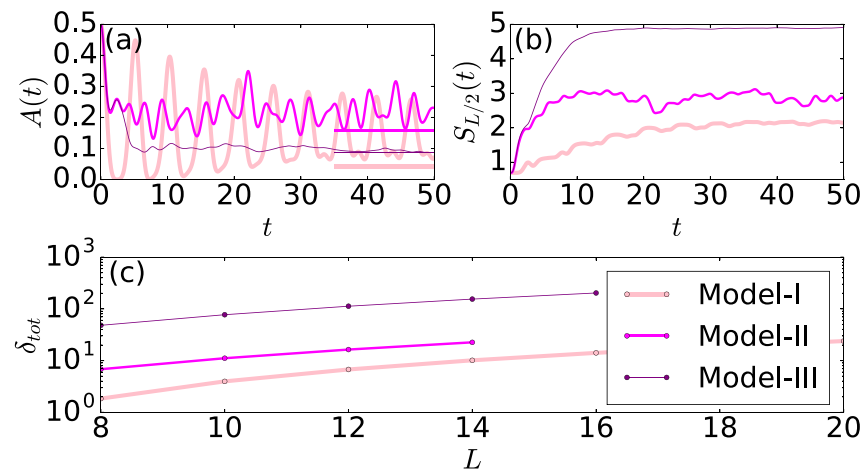

FIG. 5. (a) Dynamics of a local observable (see text) in the three models starting from an inversion symmetric version of $\left|\mathbb{Z}_{2}\right\rangle$. Thermal (infinite temperature) values are shown by horizontal lines at bottom right corner, $L=12$. (b) Dynamics of $S_{L / 2}, L=12$. (c) Behavior of total FSA errors as a function of system size. 
error free) and error arises at fifth $\left(n_{f}\right)$ step. For model II and III error arises at third and second FSA step, respectively. This errors cause dephasing; higher the errors, higher is the rate of dephasing. Here we give analytical expression (see Appendix D) of first nonzero FSA error $\left(\delta_{n_{f}}^{\alpha}\right)$ for the three models

$$
\begin{aligned}
& \delta_{n_{f}(=5)}^{I}=\frac{12\left(L^{3}-6 L^{2}+11 L-18\right)}{(L-1)(L-2)(L-3)\left(5 L^{4}-50 L^{3}+175 L^{2}-250 L+144\right)} \\
& \delta_{n_{f}(=3)}^{\mathrm{II}}=\frac{50(2 L-9)}{(2 L-5)\left(6 L^{2}-45 L+95\right)} \\
& \delta_{n_{f}(=2)}^{\mathrm{III}}=\frac{1}{4(4 L-11)} .
\end{aligned}
$$

In brief, $\delta_{n_{f}} \sim L^{-\left(n_{f}-1\right)}$ at large $L$, from which it is easy to see that $\delta_{n_{f}}^{\mathrm{I}}<\delta_{n_{f}}^{\mathrm{II}}<\delta_{n_{f}}^{\mathrm{III}}$. We numerically calculate the FSA errors in higher steps $\left(n>n_{f}\right)$ and plot the behavior of total FSA error $\left(\delta_{\text {tot }}=\sum_{n=1}^{2 L} \delta_{n}\right)$ in Fig. 5. One can again see that $\delta_{\text {tot }}^{\text {I }}<\delta_{\text {tot }}^{\text {II }}<\delta_{\text {tot }}^{\text {III }}$ The huge total FSA error of model III is not an artifact of the $\mathbb{Z}_{2}$ initial state but true for other kind of starting states also. This gives a qualitative understanding of the hierarchy of entanglement growth, amount of oscillation present in the dynamics of local observable and ergodicity of the models.

\section{STABILITY AGAINST PERTURBATION}

In this section we discuss the stability of the scars and the corresponding nonthermal dynamics in model I and II as well as the fate of the thermal dynamics of model III against the following two perturbations:

$$
\begin{aligned}
& \delta H_{1}^{\alpha}=\lambda \sum_{i} S_{i}^{z} \\
& \delta H_{2}^{\alpha}=h_{x z} \sum_{i} \mathcal{P}^{\alpha} S_{i}^{x} \mathcal{P}^{\alpha}\left(S_{i-2}^{z}+S_{i+2}^{z}\right),
\end{aligned}
$$

the former perturbation can be regarded as a uniform chemical potential term which breaks the particle-hole $(E \rightarrow-E)$ symmetry and is known to destroy the scar-induced nonergodicity in spin-1/2 PXP model [15]. This term, being diagonal in $S^{z}$ basis, does not change the fragmentation structure of model I. Therefore the fate of the special states (residing in small fragments) with integer energies under this perturbation can be studied exactly. We find, though the energy and magneti- zation of the special states are sensitive to this perturbation, interestingly the structure of the reduced density matrices and hence entanglement of the states remain unchanged at any $\lambda$. This is due to the localized structure of these states which are mostly inert except a few active sites separated by wide frozen region, consequently mixing of states by $\delta H_{1}^{\mathrm{I}}$ is not sufficient to increase entanglement. The completely inert states continues to remain exact eigenstates of the whole Hamiltonian with only shifting their energies to some nonzero values. On the other hand, eigenstates in the larger fragments are strongly mixed among themselves by $\delta H_{1}^{\mathrm{I}}$, leading to the destruction of scar states. This, in turn leads to rapid growth of entanglement starting from local initial state like $\left|\mathbb{Z}_{2}\right\rangle$. For model II, all the local conserved quantities $\left(O_{i} \mathrm{~s}\right)$ are destroyed at nonzero $\lambda$. Though, the conserved quantities may remain approximately conserved at very small $\lambda$, strong $\lambda$ thermalizes these local objects as well as the entanglement (see Fig. 6). Model-III which has not any scars and already ergodic, does not suffer from any appreciable change of ergodicity under $\delta H_{1}^{\mathrm{III}}$.

$\delta H_{2}^{\alpha}$ is a PXP term whose sign depends on the state of the next-nearest-neighbor spin. This term keeps the particle-hole symmetry intact and also does not change the fragmentation structure of model I. For the special states, it only changes their energy but the eigenstates remain unchanged. Consequently expectation value of any operator as well as the entanglement of the special states remain conserved under $\delta H_{2}^{\mathrm{I}}$. This term is known to have the potential of stabilizing the scars in spin-1/2 PXP model by reducing the FSA errors to zero which leads to near perfect $\mathbb{Z}_{2}$ oscillation via the emergence of an SU(2) algebra [19]. Unfortunately, in the spin-1 PXP models considered in this work, it increases the
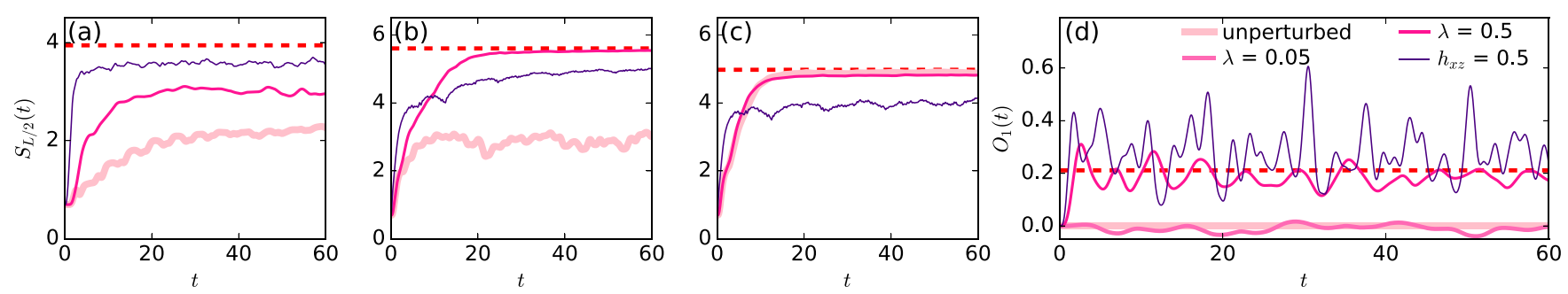

FIG. 6. Change of entanglement growth rate in perturbed model I (a), model II (b), and model III (c) starting from the inversion symmetric version of $\left|\mathbb{Z}_{2}\right\rangle$ for $L=12$. Dynamics of $O_{1}$ (which is a local conserved quantity for unperturbed model II) from $\left|\mathbb{Z}_{2}\right\rangle$ by the perturbed model II under different perturbation strength is shown in (d) for $L=8$. Page value of $S_{L / 2}$ and thermal value of $O_{1}$ is shown by red dashed lines. 
FSA errors. For example, in presence of nonzero $h_{x z}$, FSA error in model I now arises at third step instead of fifth step, and at second step instead of third step for model II. This contributes to the huge increase of $\delta_{\text {tot }}$ with $h_{x z}$ which causes strong mixing of the eigenstates in larger fragments and leads to more rapid growth of entanglement compared to what is done by $\delta H_{1}^{\mathrm{I}}$ for model I. Non-zero $h_{x z}$ also destroy the conserved quantities of model II which no longer can be decomposed into spin-1/2 PXP blocks of different sizes. Large $h_{x z}$ causes strong mixing also for model II which again leads to more rapid growth of entanglement compared to the unperturbed case. Finally, model III being strongly ergodic at the beginning, saturates to a relatively low entangled state under strong $\delta H_{2}^{\mathrm{III}}$.

\section{CONCLUSION AND DISCUSSION}

In summary we have studied the change in ergodic properties of spin-1 PXP model for three specific choices of constraints. We find that whereas certain set of constraints can strongly shatter the Hilbert space into exponentially many emergent subsectors, thus leading to violation of ETH, some other set of constraints can destroy all the anomalous states and make the spectrum strongly ergodic. Many other set of constraints can be designed between the excited levels of nearest-neighbor atoms. A detail study of all such set of constraints is beyond the scope of the current work [27]. But we have checked that there are other set of constraints which belong to the first category (i.e., in the class of model I). For example, if only $|+0\rangle$ and $|0+\rangle$ type of configurations are disallowed, then also the constrained Hilbert space $\left(d_{L}^{\mathrm{OBC}} \sim 2.414^{L}\right)$ gets shattered into many blocks of different sizes. In fact the special eigenstates of model I (with integer energies) are also the eigenstates of this model. Note that this model differs from model I by only the absence of $|00\rangle$ type of configurations which means the inert sector or the special eigenstates of model I are robust against this change of constraints. On the experimental side, we note that nonergodic quantum dynamics due to emergent kinetic constraint and Hilbert space fragmentation is recently observed in tilted Fermi-Hubbard model at large tilt potential [56]. Experimental realization of spin-1 PXP models needs three level atoms. Two different Rydberg excited state [57] will be necessary for this purpose as hardcore constraints are imposed typically between the first and second excited levels of neighboring atoms. Second, we believe that the model II is a minimal interacting model where nonintegrability and extensive number of local conserved quantities coexist. It will be interesting to explore the existence of such models in other type of systems [54]. Finally, the strong ergodic nature of model III is also remarkable because we find that quantum scars exist and ETH violation happens even when only $|++\rangle$ type of configurations are forbidden (results are not presented here). This phenomenon is quite amazing as according to the current consensus ETH is expected to be compromised to some extent if the system has kinetic constraint. We leave the detailed mechanism behind this constraint induced strong ergodicity and the exploration of class of constraints which leads to the same behavior as an interesting future problem.

\section{ACKNOWLEDGMENTS}

This work is supported by the Shanghai Municipal Science and Technology Major Project (Grant No. 2019SHZDZX01) (B.M., Z.C., and W.V.L.), National Key Research and Development Program of China (Grants No. 2016YFA0302001 and No. 2020YFA0309000) and NSFC of China (Grants No. 11674221 and No. 11574200) (Z.C.) and by the AFOSR Grant No. FA9550-16-1-0006 and the MURI-ARO Grant No. W911NF17-1-0323 through UCSB (W.V.L.). B.M. thanks Arnab Sen, Debasish Banerjee, Krishnendu Sengupta, Sayan Choudhury and Sthitadhi Roy for stimulating discussions.

\section{APPENDIX A: GROWTH OF HILBERT SPACE DIMENSION FOR MODELS I, II, AND III}

The constraints leads to a slower growth of Hilbert space dimension compared to naive $3^{L}$. We demonstrate it for the OBC. We start by model I for which $|+0\rangle,|0+\rangle$, and $|00\rangle$ type of configurations are not allowed. Any state in a system of size $L$ may end by $|-\rangle$, $|0\rangle$, or $|+\rangle$. All states in a $L$ site system which are ending by $|-\rangle$ can be obtained by simply appending a $|-\rangle$ to all states in a $L-1$ site system. The states which are ending by $|0\rangle$ in a $L$ site system can be obtained by appending $|-0\rangle$ to all states in a $L-2$ site system, whereas $|+\rangle$ can only be appended if the last site is not in the state $|0\rangle$. This leads to the following recurrence relation of total number of states $\left(d_{L}\right)$ in a system of size $L$ :

$$
\begin{aligned}
d_{L} & =d_{L}^{-}+d_{L}^{0}+d_{L}^{+} \\
& =d_{L-1}+d_{L-2}+\left(d_{L-1}-d_{L-3}\right) \\
& =2 d_{L-1}+d_{L-2}-d_{L-3},
\end{aligned}
$$

which can be cast in the following matrix form:

$$
\left(\begin{array}{c}
d_{L} \\
d_{L-1} \\
d_{L-2}
\end{array}\right)=\left(\begin{array}{ccc}
2 & 1 & -1 \\
1 & 0 & 0 \\
0 & 1 & 0
\end{array}\right)\left(\begin{array}{l}
d_{L-1} \\
d_{L-2} \\
d_{L-3}
\end{array}\right)
$$

The eigenvalues of this matrix are $\approx(2.247,-0.802,0.555)$. It is difficult to get an exact expression of $d_{L}$ for model I but the leading behavior in the large $L$ limit will be controlled by the largest eigenvalue 2.247 (as this is the only one with magnitude $>1$ ).

Model II does not allow $|00\rangle$ type configurations only. So $|0\rangle$ can only be appended if the last site is either in state $|+\rangle$ or $|-\rangle$. The recurrence relation for $d_{L}$ is given by

$$
\begin{aligned}
d_{L} & =d_{L}^{-}+d_{L}^{0}+d_{L}^{+} \\
& =d_{L-1}+\left(d_{L-1}^{+}+d_{L-1}^{-}\right)+d_{L-1} \\
& =2 d_{L-1}+2 d_{L-2},
\end{aligned}
$$

which can be cast in the following matrix form:

$$
\left(\begin{array}{c}
d_{L} \\
d_{L-1}
\end{array}\right)=\left(\begin{array}{ll}
2 & 2 \\
1 & 0
\end{array}\right)\left(\begin{array}{l}
d_{L-1} \\
d_{L-2}
\end{array}\right)=\left(\begin{array}{ll}
2 & 2 \\
1 & 0
\end{array}\right)^{L-2}\left(\begin{array}{l}
d_{2} \\
d_{1}
\end{array}\right)
$$


using $d_{1}=3$ and $d_{2}=8$ one get the expression of $d_{L}$ as a function of $L$ (see Table I in main text).

In model III $|+0\rangle /|0+\rangle$ type of configurations are not allowed. States which are ending by $|0\rangle(|+\rangle)$ in a $L$ site system can be obtained by appending $|0\rangle(|+\rangle)$ to all states of a $L-1$ site system which are not ending by $|+\rangle(|0\rangle)$. Therefore,

$$
\begin{aligned}
d_{L} & =d_{L}^{-}+d_{L}^{0}+d_{L}^{+} \\
& =d_{L-1}+\left(d_{L-1}-d_{L-1}^{+}\right)+\left(d_{L-1}-d_{L-1}^{0}\right) \\
& =3 d_{L-1}-\left(d_{L-1}^{0}+d_{L-1}^{+}\right) \\
& =2 d_{L-1}+d_{L-2}
\end{aligned}
$$

and this recurrence relation can be represented in the following matrix form:

$$
\left(\begin{array}{c}
d_{L} \\
d_{L-1}
\end{array}\right)=\left(\begin{array}{ll}
2 & 1 \\
1 & 0
\end{array}\right)\left(\begin{array}{l}
d_{L-1} \\
d_{L-2}
\end{array}\right)=\left(\begin{array}{ll}
2 & 1 \\
1 & 0
\end{array}\right)^{L-2}\left(\begin{array}{l}
d_{2} \\
d_{1}
\end{array}\right)
$$

and the exact expression of $d_{L}$ for model I can be calculated using $d_{1}=3$ and $d_{2}=7$ (see Table I in main text).

Hilbert space dimension in the periodic boundary condition PBC will be somewhat smaller than the corresponding number in $\mathrm{OBC}$ as some of the configurations (which does not satisfies the constraints between the two end spins) will be eliminated.

\section{APPENDIX B: SPECIAL STATES OF MODEL I AND THEIR PROPERTIES}

\section{Inert states}

There are exponentially many states inside the constrained Hilbert space of model I that are not connected by the Hamiltonian with other states and hence inert $[18,50]$. Inert states are unentangled, zero-energy eigenstates of $H^{I}$. We first consider the most obvious one: $|+++\cdots\rangle$, the product of $\left|S_{z}=1\right\rangle$ state at all sites. Starting from this state, one can construct other inert states by inserting one or more $|-\rangle$ in the sea of $|+\rangle$ states. There can not be three consecutive $|-\rangle$ state and isolated (i.e., surrounded by at least one $|-\rangle$ from both side) $|+\rangle$ state, as the presence of such configurations makes the state active. Note that, due to the same reason, presence of any |0) state is also not allowed. We find that the exact number of inert states $\left(I_{L}\right)$ for system size $L$ follows the recurrence relation $I_{L}=I_{L-1}+I_{L-3}+I_{L-4}$ which can be cast in the following matrix form:

$$
\left(\begin{array}{c}
I_{L} \\
I_{L-1} \\
I_{L-2} \\
I_{L-3}
\end{array}\right)=\left(\begin{array}{llll}
1 & 0 & 1 & 1 \\
1 & 0 & 0 & 0 \\
0 & 1 & 0 & 0 \\
0 & 0 & 1 & 0
\end{array}\right)\left(\begin{array}{c}
I_{L-1} \\
I_{L-2} \\
I_{L-3} \\
I_{L-4}
\end{array}\right) .
$$

Interestingly this holds for both $\mathrm{PBC}$ and $\mathrm{OBC}$. Therefore, raising this matrix to appropriate power and using suitable boundary conditions we get

$$
\begin{aligned}
I_{L}^{\mathrm{OBC}}= & \frac{1}{10}\left\{(3+\sqrt{5}) \phi^{L}-(3-\sqrt{5})\left(\frac{-1}{\phi}\right)^{L}\right. \\
& \left.+4\left[\cos \left(\frac{\pi L}{2}\right)-2 \sin \left(\frac{\pi L}{2}\right)\right]\right\}
\end{aligned}
$$

$$
I_{L}^{\mathrm{PBC}}=2 \cos \left(\frac{\pi L}{2}\right)+\phi^{L}+\phi^{-L},
$$

where $\phi\left(=\frac{1+\sqrt{5}}{2}\right)$ is the golden ratio. It is easy to see that for large systems $I_{L} \sim c \phi^{L}$ where $c=1(0.523)$ for PBC(OBC) which means there is nearly half amount of inert states in OBC compared to PBC.

\section{Special states with integer energies}

Special states with energy $E= \pm 1$ are given by

$$
\left|\psi_{ \pm 1}\right\rangle=\frac{1}{2}\left|\psi_{-}\right\rangle+\frac{1}{2}\left|\psi_{+}\right\rangle \pm \frac{1}{\sqrt{2}}\left|\psi_{0}\right\rangle
$$

where

$$
\begin{aligned}
& \left|\psi_{-}\right\rangle=\frac{1}{\sqrt{L}} \sum_{n=1}^{L} T^{n}|\underbrace{++\cdots++}_{L-3}---\rangle \\
& \left|\psi_{+}\right\rangle=\frac{1}{\sqrt{L}} \sum_{n=1}^{L} T^{n}|\underbrace{++\cdots++}_{L-3}-+-\rangle \\
& \left|\psi_{0}\right\rangle=\frac{1}{\sqrt{L}} \sum_{n=1}^{L} T^{n}|\underbrace{++\cdots++}_{L-3}-\mathbf{0}-\rangle .
\end{aligned}
$$

Next, eigenstates with energy $E= \pm 2$ are given by

$$
\begin{aligned}
\left|\psi_{ \pm 2}\right\rangle= & \pm \frac{1}{4}\left|\psi_{--}\right\rangle \pm \frac{1}{\sqrt{8}}\left|\psi_{+-}\right\rangle \pm \frac{1}{4}\left|\psi_{++}\right\rangle+\frac{1}{2}\left|\psi_{0-}\right\rangle \\
& +\frac{1}{2}\left|\psi_{+0}\right\rangle \pm \frac{1}{2}\left|\psi_{00}\right\rangle,
\end{aligned}
$$

where

$$
\begin{aligned}
& \left|\psi_{--}\right\rangle=\sqrt{\frac{2}{L}} \sum_{n=1}^{L / 2} T^{n}|\underbrace{+++\cdots++}_{\frac{L}{2}-3}---\underbrace{++\cdots++}_{\frac{L}{2}-3}---\rangle \\
& \left|\psi_{+-}\right\rangle=\frac{1}{\sqrt{L}} \sum_{n=1}^{L} T^{n}|\underbrace{++\cdots++}_{\frac{L}{2}-3}-+-\underbrace{++\cdots+++}_{\frac{L}{2}-3}--\rangle \\
& \left|\psi_{++}\right\rangle=\sqrt{\frac{2}{L}} \sum_{n=1}^{L / 2} T^{n}|\underbrace{++\cdots++}_{\frac{L}{2}-3}+-\underbrace{++\cdots+++}_{\frac{L}{2}-3}-+\rangle \\
& \left|\psi_{0-}\right\rangle=\frac{1}{\sqrt{L}} \sum_{n=1}^{L} T^{n}|\underbrace{++\cdots++}_{\frac{L}{2}-3}-\mathbf{0}-\underbrace{++\cdots++}_{\frac{L}{2}-3}---\rangle \\
& \left|\psi_{+0}\right\rangle=\frac{1}{\sqrt{L}} \sum_{n=1}^{L} T^{n}|\underbrace{++\cdots++}_{\frac{L}{2}-3}-+-\underbrace{++\cdots++}_{\frac{L}{2}-3}-\mathbf{0}-\rangle \\
& \left|\psi_{00}\right\rangle=\sqrt{\frac{2}{L}} \sum_{n=1}^{L / 2} T^{n}|\underbrace{++\cdots++}_{\frac{L}{2}-3}-\mathbf{0}-\underbrace{++\cdots++}_{\frac{L}{2}-3}-\mathbf{0}-\rangle .
\end{aligned}
$$

Red (blue bold) colored sites are inert (active). Note that there is a structural resemblance between the states in Eqs. (B3) and (B4) and in Eqs. (B5) and (B6). The later type of 
states (in a system of size $L$ ) is obtained by a spatial addition of different combinations of the former type of states (in a system of size $L / 2$ ). This also explains the additivity of their energies.

\section{a. Proof of eigenstates}

Here we prove that the special states in Eqs. (B3) and (B5) are exact eigenstates of model I. We find that the following relations hold for the states in Eq. (B4) and model I:

$$
\begin{aligned}
H^{\mathrm{I}}\left|\psi_{-}\right\rangle & =\frac{1}{\sqrt{2}}\left|\psi_{0}\right\rangle \\
H^{\mathrm{I}}\left|\psi_{+}\right\rangle & =\frac{1}{\sqrt{2}}\left|\psi_{0}\right\rangle \\
H^{\mathrm{I}}\left|\psi_{0}\right\rangle & =\frac{1}{\sqrt{2}}\left(\left|\psi_{+}\right\rangle+\left|\psi_{-}\right\rangle\right) .
\end{aligned}
$$

These relations together gives $H^{\mathrm{I}}\left|\psi_{ \pm 1}\right\rangle= \pm\left|\psi_{ \pm 1}\right\rangle$.

Similarly for the states in Eq. (B6), we find

$$
\begin{aligned}
H^{\mathrm{I}}\left|\psi_{--}\right\rangle & =\left|\psi_{0-}\right\rangle \\
H^{\mathrm{I}}\left|\psi_{+-}\right\rangle & =\frac{1}{\sqrt{2}}\left(\left|\psi_{0-}\right\rangle+\left|\psi_{+0}\right\rangle\right) \\
H^{\mathrm{I}}\left|\psi_{++}\right\rangle & =\left|\psi_{+0}\right\rangle \\
H^{\mathrm{I}}\left|\psi_{0-}\right\rangle & =\left|\psi_{--}\right\rangle+\left|\psi_{00}\right\rangle+\frac{1}{\sqrt{2}}\left|\psi_{+-}\right\rangle \\
H^{\mathrm{I}}\left|\psi_{+0}\right\rangle & =\frac{1}{\sqrt{2}}\left|\psi_{+-}\right\rangle+\left|\psi_{++}\right\rangle+\left|\psi_{00}\right\rangle \\
H^{\mathrm{I}}\left|\psi_{00}\right\rangle & =\left|\psi_{0-}\right\rangle+\left|\psi_{+0}\right\rangle .
\end{aligned}
$$

These relations together gives $H^{\mathrm{I}}\left|\psi_{ \pm 2}\right\rangle= \pm 2\left|\psi_{ \pm 2}\right\rangle$.

\section{b. Magnetization}

Here we show that the special eigenstates have integer magnetization $\left(S_{z}=\sum_{i} S_{i}^{z}\right)$.

For states in Eq. (B3)

$$
\left\langle\psi_{ \pm 1}\left|S_{z}\right| \psi_{ \pm 1}\right\rangle=\frac{L-6}{4}+\frac{L-4}{4}+\frac{L-5}{2}=L-5 .
$$

For states in Eq. (B5)

$$
\begin{aligned}
\left\langle\psi_{ \pm 2}\left|S_{z}\right| \psi_{ \pm 2}\right\rangle= & \frac{L-12}{16}+\frac{L-10}{8}+\frac{L-8}{16}+\frac{L-11}{4} \\
& +\frac{L-9}{4}+\frac{L-10}{4}=L-10 .
\end{aligned}
$$

Similarly for states with energy $E= \pm n$, magnetization will be $L-5 n$. We note that there are lots of eigenstates at integer energies in model I. The specialty of integer energy eigenstates [e.g., in (B3) and (B5)] studied in this work is that they have minimum entanglement entropy and maximum magnetization in the corresponding manifold of states.

\section{c. Entanglement entropies}

The entanglement of a state can be quantified using various schemes. We use Von-Neumann formula which works in the
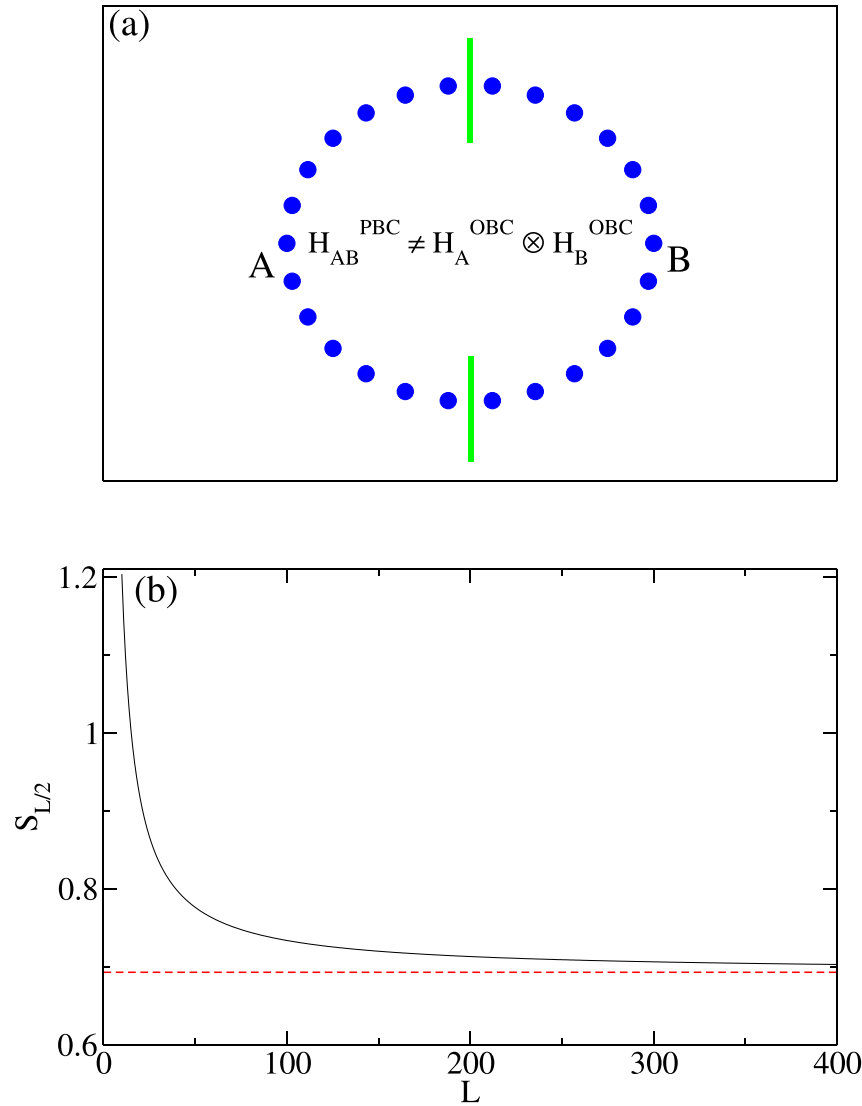

FIG. 7. (a) Partition of a constrained system into two parts. (b) $S_{L / 2}$ as a function of $L$ for the states in (B3)

following way: First, divide the full system $A B$ into two parts, $A$ and $B$. Then the entropy of entanglement of part $A$ with part $B$ is given by $S_{A}=\operatorname{Tr}\left[\rho_{A} \ln \left(\rho_{A}\right)\right]$, where $\rho_{A}=\operatorname{Tr}_{B}\left[\rho_{A B}\right]$ is the reduced density matrix of part $A$ and $\rho_{A B}$ is the density matrix corresponding to the state of the full system. The size of $A$ and $B$ can be anywhere in between 1 and $L-1$ with the constraint $L_{A}+L_{B}=1$. As we use PBC for all our calculation, the full system $A B$ will be in $\mathrm{PBC}$ we have to treat the individual part $A$ and $B$ using OBC. One needs to be careful while taking the partial trace of the full density matrix (to ensure $\operatorname{Tr}\left[\rho_{A}\right]=1$ ) as due to the constraints in Hilbert space, the state of $A B$ is not tensor product of states in $A$ and $B$ (see Fig. 7).

We first derive the single site reduced density matrices $\left(\rho_{1}\right)$ [58] for the special states in Eq. (B3). The local HSD per site is 3 , so $\rho_{1}$ is a $3 \times 3$ matrix, whose diagonal elements for the states in Eq. (B3) are

$$
\begin{aligned}
\rho_{1}^{++} & =\frac{1}{4} \frac{1}{L}(L-3)+\frac{1}{4} \frac{1}{L}(L-2)+\frac{1}{2} \frac{1}{L}(L-3) \\
& =1-\frac{11}{4 L} \\
\rho_{1}^{00} & =\frac{1}{2} \frac{1}{L} \\
\rho_{1}^{--} & =\frac{1}{4} \frac{1}{L} 3+\frac{1}{4} \frac{1}{L} 2+\frac{1}{2} \frac{1}{L} 2=\frac{9}{4 L} .
\end{aligned}
$$



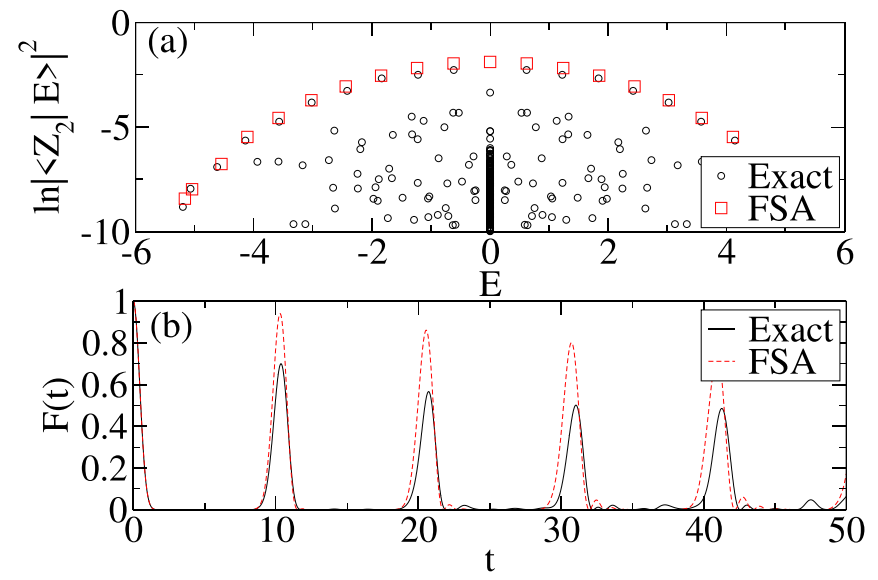

FIG. 8. Comparison of FSA with exact numerics for model I: (a) $\mathbb{Z}_{2}$ overlap and (b) Fidelity dynamics of the $\mathbb{Z}_{2}$ state. $L=10$.

The off-diagonal elements are

$$
\begin{aligned}
& \rho_{1}^{+0}=\rho_{1}^{0+}=\frac{1}{2} \frac{1}{\sqrt{2}} \frac{1}{L} \\
& \rho_{1}^{+-}=\rho_{1}^{-+}=\frac{1}{2} \frac{1}{2} \frac{1}{L} \\
& \rho_{1}^{0-}=\rho_{1}^{-0}=\frac{1}{2} \frac{1}{\sqrt{2}} \frac{1}{L} .
\end{aligned}
$$

Note that all matrix elements except one diagonal element die out in the thermodynamic limit which means $\lim _{L \rightarrow \infty} S_{1}=0$. Therefore, in the thermodynamic limit, any site is unentangled with the rest of the system. This holds for the state in Eq. (B5) also.
Now we concentrate on half chain entanglement entropy. Though the dimension of the corresponding reduced density matrices $\left(\rho_{L / 2}\right)$ scales as $\approx 2.249^{L / 2}$, for the states in Eq. (B3) we find only six eigenvalues are nonzero at any system size $L$, which are $\frac{L-4}{2 L}$ (multiplicity 2) and $\frac{1}{L}$ (multiplicity 4). This gives $S_{L / 2}=-2 \frac{L-4}{2 L} \ln \left(\frac{L-4}{2 L}\right)-4 \frac{1}{L} \ln \left(\frac{1}{L}\right)$ which has been plotted in Fig. 7. The decrease of $S_{L / 2}$ with $L$ and its saturation to the area law value $(\ln 2)$ in asymptotically large system size is an artifact of the nontensor-product structure of the constrained Hilbert space. For the states in Eq. (B5) we find the number of nonzero eigenvalues to be $L / 2$ which are all equal with magnitude $\frac{2}{L}$. This gives $S_{L / 2}=\frac{L}{2} \frac{2}{L} \ln \left(\frac{2}{L}\right)=$ $\ln (L)-\ln (2)$.

\section{APPENDIX C: CONSERVED QUANTITIES OF MODEL II}

Here we show that the operators $O_{i}$ commutes with the Hamiltonian $H^{\mathrm{II}}$. We expand the relevant portion of $H^{\mathrm{II}}$,

$$
\mathcal{P}_{i-1, i}^{\mathrm{II}} S_{i}^{x} \mathcal{P}_{i, i+1}^{\mathrm{II}}=\mathbb{I}_{i-1} \otimes S_{i}^{x} \otimes \mathbb{I}_{i+1}-\frac{1}{\sqrt{2}} \mathbb{I}_{i-1} \otimes(|+\rangle\langle 0|+|-\rangle\langle 0|)_{i} \otimes(|0\rangle\langle 0|)_{i+1}-\frac{1}{\sqrt{2}}(|0\rangle\langle 0|)_{i-1} \otimes(|0\rangle\langle+|+| 0\rangle\langle-|)_{i} \otimes \mathbb{I}_{i+1}
$$

where we have used that $S_{i}^{x}=\frac{1}{\sqrt{2}}(|+\rangle\langle 0|+| 0\rangle\langle+|+| 0\rangle\langle-|+|-\rangle\langle 0|)_{i}$ and $\mathcal{P}_{i, i+1}^{\text {II }}=\mathbb{I}_{i} \otimes \mathbb{I}_{i+1}-(|0\rangle\langle 0|)_{i} \otimes(|0\rangle\langle 0|)_{i+1}$. It is easy to see that each part of Eq. (C1) individually commutes with $O_{i}(=|+\rangle\langle-|+| 0\rangle\langle 0|+|-\rangle\langle+|)_{i}, O_{i-1}, O_{i+1}$. Hence, $\left[H^{\mathrm{II}}, O_{i}\right]=0, \forall i$.

\section{APPENDIX D: FORWARD SCATTERING APPROXIMATION}

FSA has been an important tool to analyze the scar induced oscillation since its first usage in spin-1/2 PXP model [14]. Here we will apply FSA in detail in our spin-1 models [27]. The essential idea is to first break the model Hamiltonians in $H_{+}$and $H_{-}$ (one is conjugate transpose to other) such that one (let us say $H_{-}$) annihilate the state $\left|\mathbb{Z}_{2}\right\rangle$ and the other $\left(H_{+}\right)$annihilate $\left|\overline{\mathbb{Z}}_{2}\right\rangle$. One state $\left(\left|\overline{\mathbb{Z}}_{2}\right\rangle /\left|\mathbb{Z}_{2}\right\rangle\right)$ can be obtained from the other $\left(\left|\mathbb{Z}_{2}\right\rangle /\left|\overline{\mathbb{Z}}_{2}\right\rangle\right)$ by repeated action $(2 L$ times $)$ of $\left(H_{+} / H_{-}\right)$. The oscillatory dynamics then can be visualized as a coherent forward and backward scattering in between these two states.

We define the $n$th FSA vector $\left(\left|v_{n}\right\rangle\right)$ by $\left|v_{n}\right\rangle=\frac{1}{\beta_{n}} H_{+}\left|v_{n-1}\right\rangle$, where $\left|v_{0}\right\rangle=\left|\mathbb{Z}_{2}\right\rangle$ and $\beta_{n}$ is the normalization constant. Due to the choice of the initial state and structure of the Hamiltonian, FSA vectors form a closed orthonormal subspace of dimension $2 L+1$. Representation of the Hamiltonian in this subspace forms a tridiagonal matrix: $H_{\mathrm{FSA}}=\beta_{n} \sum_{n=1}^{2 L}\left|v_{n}\right\rangle\left\langle v_{n+1}\right|+$ H.c. Thus, if one is interested only in the scar subspace, then an enormous simplification can be achieved, namely, one need to diagonalize a matrix whose dimension scales only linearly with system size. This enables to deal with larger system size. In Fig. 8 we have compared the $\mathbb{Z}_{2}$ overlap of the eigenstates of $H_{\mathrm{FSA}}$ and fidelity dynamics of the $\mathbb{Z}_{2}$ state generated by $H_{\mathrm{FSA}}$ with the same quantities obtained by the full Hamiltonian $\left(H^{\mathrm{I}}\right)$ for model I. To calculate other quantities (e.g., some observables, entanglement entropy, etc.) one needs to store the FSA vectors which again consumes exponential memory (though this scales as $\sim L e^{L}$ but not 
as $\sim e^{2 L}$ ). The mismatch of the FSA results with exact numerics in Fig. 8 is due to the fact that the FSA vectors does not form a complete set and hence cannot span the Hilbert space, that is why the leakage of the $\mathbb{Z}_{2}$ dynamics out of the FSA manifold is inevitable. This causes the revival amplitude to decrease and dephase the dynamics. This can be quantified using the FSA errors: $\delta_{n}=\| H_{-}\left|v_{n}\right\rangle-\beta_{n}\left|v_{n-1}\right\rangle \|$. If the action of $H_{-}$on a FSA vector completely undo the action of $H_{+}$on the same vector, then $\delta$ is zero and the corresponding FSA step is exact. In an ideal paramagnet (described by $H=\sum_{i} S_{i}^{x}$ ) all FSA steps are exact but due to the constraints induced by the projectors, FSA errors are nonzero in PXP models. Interestingly suitable term can be added to the bare PXP model which can reduce [31] the FSA errors even to zero [19]. In this situation the $\mathbb{Z}_{2}$ dynamics remains totally confined in the scar manifold which results in an undamped oscillation up to very long time. This suggests that the FSA errors produces some kind of frictional force in the system and hence the amplitude of oscillation (degree of fidelity revival) should be inversely proportional to the total (summed over all the FSA steps) amount of FSA errors present in the system. Motivated by these arguments, we plan to perform a detail analysis of the FSA errors for models I-III.

\section{FSA for model I}

In model I $|00\rangle,|+0\rangle,|0+\rangle$ type of configurations are not allowed. We start with

$$
H_{+}^{I}\left|v_{0}\right\rangle=\frac{1}{\sqrt{2}} \sum_{i \text { even }}\left|\cdots{ }_{i}^{0} \cdots\right\rangle,
$$

where $\cdots$ represents sea of repeated +- or -+ configurations (with a suitably added + or - at the end). So $\beta_{1}=\sqrt{\| H_{+}^{I}\left|v_{0}\right\rangle \|}=$ $\sqrt{L} / 2$,

$$
\left|v_{1}\right\rangle=\frac{1}{\beta_{1}} H_{+}^{I}\left|v_{0}\right\rangle=\sqrt{\frac{2}{L}} \sum_{i \text { even }}|\cdots \underset{i}{0} \cdots\rangle .
$$

One can easily check: $H_{-}^{I}\left|v_{1}\right\rangle=\beta_{1}\left|v_{0}\right\rangle$. Therefore, $\delta_{1}^{I}=0$.

Next,

$$
H_{+}^{I}\left|v_{1}\right\rangle=\frac{1}{\sqrt{L}}\left(\sum_{i \text { even }}\left|\cdots-\frac{-}{i}-\cdots\right\rangle+2 \sum_{\substack{i, j \text { even } \\ i \neq j}}|\cdots \underset{i}{0} \cdots \underset{j}{0} \cdots\rangle\right) .
$$

There are $\frac{L}{4}\left(\frac{L}{2}-1\right)$ distinct second type of states. The second summation should be understood as a sum over only this many number of states (that is why we bring a factor 2 before it). All such summation notations in this paper avoid double counting. We get, $\beta_{2}=\sqrt{|| H_{+}^{I}\left|v_{1}\right\rangle \|}=\sqrt{\frac{1}{L} \frac{L}{2}+\frac{4}{L} \frac{L}{4}\left(\frac{L}{2}-1\right)}=\frac{\sqrt{L-1}}{\sqrt{2}}$. and $\left|v_{2}\right\rangle=\frac{1}{\beta_{2}} H_{+}^{I}\left|v_{1}\right\rangle$. It is easy to see that $H_{-}^{I}\left|v_{2}\right\rangle=\beta_{2}\left|v_{1}\right\rangle . . \delta_{2}^{I}=0$.

Next we find

$$
H_{+}^{I}\left|v_{2}\right\rangle=\sqrt{\frac{1}{L(L-1)}}\left(3 \sum_{\substack{i, j \text { even } \\ i \neq j}}\left|\cdots 0_{i} \cdots---\cdots\right\rangle+6 \sum_{\substack{i, j, k \text { even } \\ i \neq j \neq k}}|\cdots \underbrace{0}_{i} \cdots \underbrace{0}_{j} \cdots 0_{k}^{0} \cdots\rangle\right),
$$

where the number of distinct second type of states is $\frac{1}{3 !} \frac{L}{2}\left(\frac{L}{2}-1\right)\left(\frac{L}{2}-2\right)=\frac{L(L-2)(L-4)}{48}$. Therefore, $\beta_{3}^{2}=\frac{1}{L(L-1)}\left[9 \frac{L}{2}\left(\frac{L}{2}-1\right)+\right.$ $\left.36 \frac{L(L-2)(L-4)}{48}\right]=\frac{3(L-2)}{4} \cdot\left|v_{3}\right\rangle=\frac{1}{\beta_{3}} H_{+}^{I}\left|v_{2}\right\rangle$ and we again find $H_{-}^{I}\left|v_{3}\right\rangle=\beta_{3}\left|v_{2}\right\rangle$, which means $\delta_{I}^{3}=0$.

Next we find

$$
\begin{aligned}
H_{+}^{I}\left|v_{3}\right\rangle= & \frac{\sqrt{2}}{\sqrt{3 L(L-1)(L-2)}}\left(3 \sum_{\substack{i, j \text { even } \\
i \neq j}}|\cdots---\cdots\rangle+12 \sum_{i}|\cdots \underbrace{}_{\substack{i, j, k \text { even } \\
i \neq j \neq k}} \cdots 0_{j} \cdots---\cdots\rangle\right. \\
& \left.+24 \sum_{\substack{i, j, k, l \text { even } \\
i \neq j \neq k \neq l}}\left|\cdots \underset{i}{0} \cdots \underset{j}{0} \cdots \underset{k}{0} \cdots 0_{l} \cdots\right\rangle\right) .
\end{aligned}
$$

Therefore, $\beta_{4}^{2}=\| H_{+}^{I}\left|v_{3}\right\rangle \|=\frac{2}{3 L(L-1)(L-2)}\left[36 \frac{L}{4}\left(\frac{L}{2}-1\right)+144 \frac{L(L-2)(L-4)}{16}+576 \frac{L(L-2)(L-4)(L-6)}{384}\right]=L-3$, where we have used the fact that the number of distinct second and third type of states in Eq. (D5) are $\frac{L(L-2)(L-4)}{16}$ and $\frac{L(L-2)(L-4)(L-6)}{384}$, respectively. $\left|v_{4}\right\rangle=\frac{1}{\beta_{4}} H_{+}^{I}\left|v_{3}\right\rangle$ and one can again check $\delta_{4}^{I}=0$.Note that the first part of $H_{+}^{I}$ (i.e., $\sum_{i \text { odd }} \sigma_{i}^{+}$) has null effect until now.

Finally, we arrive at the fifth step where nonzero FSA error arises for the first time. Calculations of FSA vectors become cumbersome from this step onward as both part of $H_{+}^{I}$ will now have nonzero actions. After regrouping all similar type of states 
we write a consolidated expression of the action of $H_{+}^{I}$ on $\left|v_{4}\right\rangle$,

$$
\begin{aligned}
& H_{+}^{I}\left|v_{4}\right\rangle=\frac{1}{\sqrt{3 L(L-1)(L-2)(L-3)}}\left(30 \sum_{\substack{i, j, k \text { even } \\
i \neq j \neq k}}|\cdots \underbrace{0}_{i} \cdots-\frac{-\cdots--}{j}-\cdots\rangle+6 \sum_{i \text { odd }}|\cdots--0--\cdots\rangle\right. \\
& \left.+60 \sum_{\substack{i, j, k, l \text { even } \\
i \neq j \neq k \neq l}}|\cdots \underset{i}{0} \cdots \underset{j}{0} \cdots \underset{k}{0} \cdots---\cdots\rangle+120 \sum_{\substack{i, j, k, l, m \text { even } \\
i \neq j \neq k \neq l \neq m}}|\cdots_{i}^{0} \cdots_{j}^{0} \underbrace{}_{j} \cdots \underset{k}{0} \cdots \underset{l}{0} \cdots \underset{m}{0} \cdots\rangle\right) \text {. }
\end{aligned}
$$

Counting the respective number of different states in Eq. (D6) we get

$$
\begin{aligned}
\beta_{5}^{2}= & \| H_{+}^{I}\left|v_{4}\right\rangle \| \\
= & \frac{1}{3 L(L-1)(L-2)(L-3)}\left[30^{2} \frac{L(L-2)(L-4)}{16}+36 \frac{L}{2}+60^{2} \frac{L(L-2)(L-4)(L-6)}{96}\right. \\
& \left.+120^{2} \frac{L(L-2)(L-4)(L-6)(L-8)}{3840}\right] \\
= & \frac{5 L^{4}-50 L^{3}+175 L^{2}-250 L+144}{4(L-1)(L-2)(L-3)} .
\end{aligned}
$$

The action of $H_{-}^{I}$ on $\left|v_{5}\right\rangle\left(=\frac{1}{\beta_{5}} H_{+}^{I}\left|v_{4}\right\rangle\right)$ gives after grouping same type of states together,

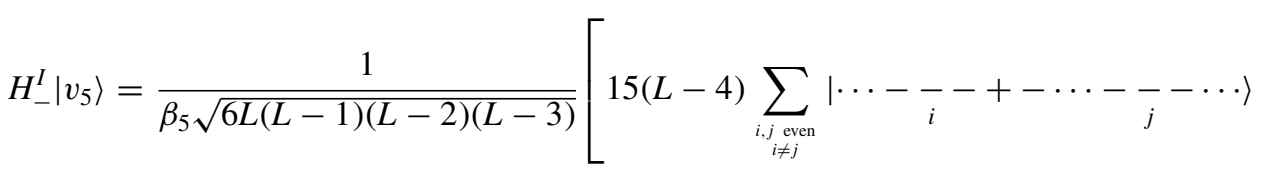

$$
\begin{aligned}
& +(15 L-54) \sum_{i \text { odd }}\left|\cdots--\frac{-}{i}-\cdots\right\rangle+30(L-4) \sum_{\substack{i, j, k \text { even } \\
i \neq j \neq k}}\left|\cdots \underset{i}{0} \cdots 0_{j} \cdots-\frac{-}{k}-\cdots\right\rangle \\
& \left.+60(L-4) \sum_{\substack{i, j, k, l \text { even } \\
i \neq j \neq k \neq l}}|\cdots \underset{i}{0} \cdots \underset{j}{0} \cdots \underset{k}{0} \cdots \underset{l}{0} \cdots\rangle\right] \text {. }
\end{aligned}
$$

Note that the first and second type of states in Eq. (D8) had same strength in $\left|v_{4}\right\rangle$ which is sufficient to see that $H_{-}^{I}\left|v_{5}\right\rangle$ is not proportional to $\left|v_{4}\right\rangle$. Thus finally error arises in fifth FSA step which can be easily calculated by evaluating the norm: $\delta_{5}^{I}=\| H_{-}^{I}\left|v_{5}\right\rangle-\beta_{5}\left|v_{4}\right\rangle \|=\frac{12\left(L^{3}-6 L^{2}+11 L-18\right)}{(L-1)(L-2)(L-3)\left(5 L^{4}-50 L^{3}+175 L^{2}-250 L+144\right)}$.

\section{FSA for model II}

Only $|00\rangle$ type of configurations are not allowed in model II. We start by

$$
H_{+}^{\mathrm{II}}\left|\mathbb{Z}_{2}\right\rangle=\frac{1}{\sqrt{2}}\left(\sum_{i \text { even }}|\cdots-\underbrace{}_{i}-\cdots\rangle+\sum_{i \text { odd }}|\cdots-+\underset{i}{0}+-\cdots\rangle\right) .
$$

So $\beta_{1}=\sqrt{|| H_{+}^{\mathrm{II}}\left|\mathbb{Z}_{2}\right\rangle \|}=\sqrt{L / 2}$ and $\left|v_{1}\right\rangle=\frac{1}{\beta_{1}} H_{+}^{\mathrm{II}}\left|\mathbb{Z}_{2}\right\rangle$. It is easy to see that $H_{-}^{\mathrm{II}}\left|v_{1}\right\rangle=\beta_{1}\left|\mathbb{Z}_{2}\right\rangle$ and hence $\delta_{1}^{\mathrm{II}}=0$. Next,

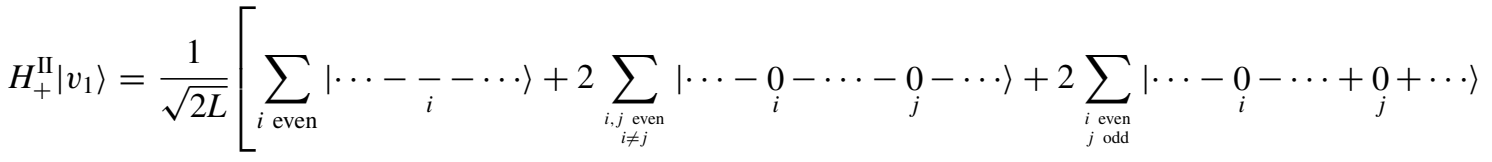

$$
\begin{aligned}
& \left.+2 \sum_{\substack{i, j \text { odd } \\
i \neq j}}|\cdots+\underbrace{}_{i}+\cdots+\underset{j}{0}+\cdots\rangle+\sum_{i \text { odd }}\left|\cdots++_{i}+\cdots\right\rangle\right]
\end{aligned}
$$


We get $\beta_{2}^{2}=\| H_{+}^{\mathrm{II}}\left|v_{1}\right\rangle \|=\frac{2 L-5}{2}$ and $\left|v_{2}\right\rangle=\frac{1}{\beta_{2}} H_{+}^{\mathrm{II}}\left|v_{1}\right\rangle$. It is easy to check that $H_{-}^{\mathrm{II}}\left|v_{2}\right\rangle=\beta_{2}\left|v_{1}\right\rangle$ which means $\delta_{2}^{\mathrm{II}}=0$. Rarity of constraints produces a large number of states in the next step, we write the consolidated expression

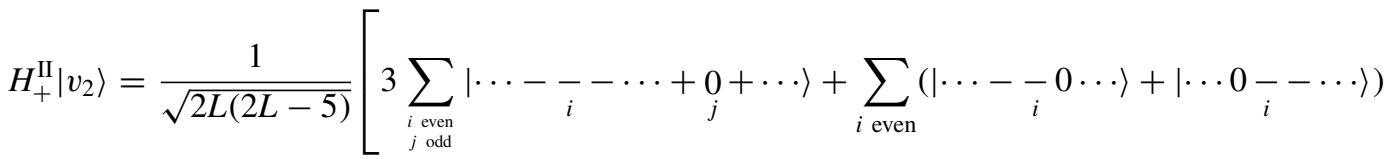

$$
\begin{aligned}
& +3 \sum_{i, j \text { even }}\left|\cdots--_{i}-\cdots-\underset{j}{0}-\cdots\right\rangle+3 \sum_{i, j \text { odd }}\left|\cdots++_{i}+\cdots+\underset{j}{0}+\cdots\right\rangle+\sum_{i \text { even }}\left(\left|\cdots++_{i} 0 \cdots\right\rangle+|\cdots 0+\cdots+\cdots\rangle\right) \\
& +3 \sum_{\substack{i \text { even } \\
j \text { odd }}}|\cdots-\underset{i}{0}-\cdots+\underset{j}{+}+\cdots\rangle+6 \sum_{\substack{i, j, k \text { even } \\
i \neq j \neq k}}\left|\cdots-0_{i}^{0}-\cdots-\underset{j}{0}-\cdots-0_{k}-\cdots\right\rangle \\
& +6 \sum_{\substack{i, j, k \text { odd } \\
i \neq j \neq k}}|\cdots+0_{i}+\cdots+\underset{j}{0}+\cdots+\underbrace{0}_{k}+\cdots\rangle+6 \sum_{\substack{i, j \text { even } \\
k \text { odd }}}|\cdots-\underbrace{}_{i}-\cdots-\underset{j}{0}-\cdots+\underset{k}{0}+\cdots\rangle \\
& \left.+6 \sum_{\substack{i, j \text { odd } \\
k \text { even }}}|\cdots+\underset{i}{0}+\cdots+\underset{j}{0}+\cdots-\underset{k}{0}-\cdots\rangle\right]
\end{aligned}
$$

by carefully counting the number of each type of states in Eq.(D11) we find

$$
\begin{aligned}
\beta_{3}^{2} & =\frac{1}{2 L(2 L-5)}\left[2\left(9 \frac{L}{2}\left(\frac{L}{2}-2\right)+L+9 \frac{L}{2}\left(\frac{L}{2}-2\right)\right)+2\left(36 \frac{L(L-2)(L-4)}{48}+36 \frac{L(L-4)(L-6)}{16}\right)\right] \\
& =\frac{6 L^{2}-45 L+95}{4 L-10}
\end{aligned}
$$

and $\left|v_{3}\right\rangle=\frac{1}{\beta_{3}} H_{+}^{\mathrm{II}}\left|v_{2}\right\rangle$. We now calculate $H_{-}^{\mathrm{II}}\left|v_{3}\right\rangle$ :

$$
\begin{aligned}
H_{-}^{\mathrm{II}}\left|v_{3}\right\rangle= & \frac{1}{\sqrt{2}} \frac{1}{\sqrt{2 L(2 L-5)}} \frac{1}{\beta_{3}}\left\{\left[3\left(\frac{L}{2}-2\right)+2+3\left(\frac{L}{2}-1\right)\right]\left(\sum_{i \text { even }}\left|\cdots--_{i}-\cdots\right\rangle+\sum_{i \text { odd }}\left|\cdots++_{i}+\cdots\right\rangle\right)\right. \\
& +\left(6+6 \frac{L-6}{2}+6 \frac{L-6}{2}\right) \sum_{\substack{i \text { even } \\
j \text { odd }}}|\cdots-\underset{i}{0}-\cdots+\underset{j}{0}+\cdots\rangle+\left(6+6 \frac{L-4}{2}+6 \frac{L-8}{2}\right) \\
& \times\left(\sum_{i, j \text { even }}|\cdots-\underset{i}{0}-\cdots+-+\cdots-\underset{j}{0}-\cdots\rangle+\sum_{i, j \text { odd }}|\cdots+\underset{i}{0}+\cdots+-+\cdots+\underset{j}{0}+\cdots\rangle\right) \\
& \left.+\left(6+6 \frac{L-4}{2}+6 \frac{L-6}{2}\right) \sum_{i \text { even }}(|\cdots-\underset{i}{0}-0-\cdots\rangle+|\cdots+\underset{i}{0}+0+\cdots\rangle)\right\},
\end{aligned}
$$

we then find that $\delta_{3}^{\mathrm{II}}=\| H_{-}^{\mathrm{II}}\left|v_{3}\right\rangle-\beta_{3}\left|v_{2}\right\rangle \|=\frac{50(2 L-9)}{(2 L-5)\left(6 L^{2}-45 L+95\right)}$.

\section{FSA for model III}

In model III the $|00\rangle$ and $|++\rangle$ type of configurations are forbidden but $|+\rangle$ and $|0\rangle$ can sit next to each other. We start by

$$
H_{+}^{\mathrm{III}}\left|\mathbb{Z}_{2}\right\rangle=\frac{1}{\sqrt{2}}\left(\sum_{i \text { even }}|\cdots-\underset{i}{0}-\cdots\rangle+\sum_{i \text { odd }}|\cdots-+\underset{i}{0}+-\cdots\rangle\right) .
$$

So $\beta_{1}=\sqrt{\| H_{+}^{\mathrm{III}}\left|\mathbb{Z}_{2}\right\rangle \|}=\sqrt{L / 2}$ and $\left|v_{1}\right\rangle=\frac{1}{\beta_{1}} H_{+}^{\mathrm{III}}\left|\mathbb{Z}_{2}\right\rangle$. We checked that $H_{-}^{\mathrm{III}}\left|v_{1}\right\rangle=\beta_{1}\left|\mathbb{Z}_{2}\right\rangle$ and hence $\delta_{1}^{\mathrm{III}}=0$.

Next

$$
\begin{aligned}
H_{+}^{\mathrm{III}}\left|v_{1}\right\rangle= & \frac{1}{\sqrt{2 L}}\left(\sum_{i \text { even }}\left|\cdots--_{i}-\cdots\right\rangle+2 \sum_{\substack{i, j \text { even } \\
i \neq j}}|\cdots-\underbrace{}_{i}-\cdots-j_{j}-\cdots\rangle+2 \sum_{\substack{i \text { even } \\
j \text { odd }}}|\cdots-\underset{i}{0}-\cdots+\underset{j}{0}+\cdots\rangle\right. \\
& \left.+2 \sum_{\substack{i, j \text { odd } \\
i \neq j}}|\cdots+\underset{i}{0}+\cdots+\underset{j}{0}+\cdots\rangle\right)
\end{aligned}
$$


counting distinct number of four different type of states in Eq. (D15), we get $\beta_{2}^{2}=\frac{1}{2 L}\left[\frac{L}{2}+4 \frac{L}{4}\left(\frac{L}{2}-1\right)+4 \frac{L}{2}\left(\frac{L}{2}-2\right)+4 \frac{L}{4}\left(\frac{L}{2}-\right.\right.$ $1)]=L-\frac{11}{4} \cdot\left|v_{2}\right\rangle=\frac{1}{\beta_{2}} H_{+}^{\mathrm{III}}\left|v_{1}\right\rangle$.

Next we find

$$
H_{-}^{\mathrm{III}}\left|v_{2}\right\rangle=\frac{1}{\sqrt{L(4 L-11)}}\left[(2 L-5) \sum_{i \text { even }}\left|\cdots-0_{i}-\cdots\right\rangle+(2 L-6) \sum_{i \text { odd }}\left|\cdots+{ }_{i}+\cdots\right\rangle\right],
$$

clearly $H_{-}^{\mathrm{III}}\left|v_{2}\right\rangle$ is not equal to $\left|v_{1}\right\rangle$ multiplied by $\beta_{2}$ and this produces error in the second FSA step. The norm of the difference is given by

$$
\delta_{2}^{\mathrm{III}}=|| H_{-}^{\mathrm{III}}\left|v_{2}\right\rangle-\beta_{2}\left|v_{1}\right\rangle \|=\frac{1}{4(4 L-11)} .
$$

\section{APPENDIX E: STABILITY OF SPECIAL STATES OF MODEL I UNDER PERTURBATIONS}

In this Appendix we provide a detailed calculation of modification of the properties of special eigenstates of model I under the perturbations in Eq. (5). We consider the fate of the special states with $E=0, \pm 1$ and $E= \pm 2$ which belong to a $3 \times 3$ and $6 \times 6$ fragment, respectively.

We first write down $H^{\mathrm{I}}+\lambda \delta H_{1}^{\mathrm{I}}$ in the $3 \times 3$ closed subspace spanned by the momentum basis vectors: $\left|\psi_{m}\right\rangle=$ $\frac{1}{\sqrt{L}} \sum_{n=1}^{L} T^{n}\left[\otimes_{i=1}^{L-3}|+\rangle_{i} \otimes|-\mathbf{m}-\rangle\right], \boldsymbol{m}= \pm, 0$ which is given by

$$
\left[\begin{array}{ccc}
\lambda(L-4) & \frac{1}{\sqrt{2}} & 0 \\
\frac{1}{\sqrt{2}} & \lambda(L-5) & \frac{1}{\sqrt{2}} \\
0 & \frac{1}{\sqrt{2}} & \lambda(L-6)
\end{array}\right] .
$$

This yields the eigenvalues: $\lambda(L-5), \lambda(L-5) \pm \sqrt{1+\lambda^{2}}$ with the corresponding unnormalized eigenvectors: $(-1, \sqrt{2} \lambda, 1)^{T}$, $\left[\left(\lambda \pm \sqrt{1+\lambda^{2}}\right)^{2}, \sqrt{2}\left(\lambda \pm \sqrt{1+\lambda^{2}}\right), 1\right]^{T}$ respectively. Using these, the magnetization of the perturbed special eigenstates are found to be $L-5, L-5 \pm \frac{\lambda}{\sqrt{1+\lambda^{2}}}$ respectively.

Next, we write down $H^{\mathrm{I}}+\lambda \delta H_{1}^{\mathrm{I}}$ in the $6 \times 6$ basis in Eq. (B6) to explore the effect of this perturbation on the $E= \pm 2$ special eigenstates. This is given by

$$
\left[\begin{array}{cccccc}
\lambda(L-12) & 0 & 0 & 1 & 0 & 0 \\
0 & \lambda(L-10) & 0 & \frac{1}{\sqrt{2}} & \frac{1}{\sqrt{2}} & 0 \\
0 & 0 & \lambda(L-8) & 0 & 1 & 0 \\
1 & \frac{1}{\sqrt{2}} & 0 & \lambda(L-11) & 0 & 1 \\
0 & \frac{1}{\sqrt{2}} & 1 & 0 & \lambda(L-9) & 1 \\
0 & 0 & 0 & 1 & 1 & \lambda(L-10)
\end{array}\right] .
$$

The eigenvalues are $\lambda(L-10)$ (multiplicity 2$), \lambda(L-10) \pm \sqrt{1+\lambda^{2}}$, and $\lambda(L-10) \pm 2 \sqrt{1+\lambda^{2}}$. The last two eigenvalues give the $E= \pm 2$ eigenvalues for $\lambda=0$. Their magnetization is found to be $L-10 \pm \frac{2 \lambda}{\sqrt{1+\lambda^{2}}}$. Note that, though in presence of $\delta H_{1}^{\mathrm{I}}$ the particle-hole symmetry is broken, the $E \rightarrow-E$ symmetry is accidentally restored at the system size where these states first appear.

Similarly, the effect of the perturbation $\delta H_{2}^{\mathrm{I}}$ on the special states can be analyzed. In this case, we find, only the energy eigenvalues are changed but wave functions remain same. Consequently, expectation value of any operator in the special states are not modified by this perturbation.

[1] L. D’Alessio, Y. Kafri, A. Polkovnikov, and M. Rigol, From quantum chaos and eigenstate thermalization to statistical mechanics and thermodynamics, Adv. Phys. 65, 239 (2016).

[2] J. M. Deutsch, Quantum statistical mechanics in a closed system, Phys. Rev. A 43, 2046 (1991).

[3] M. Srednicki, Chaos and quantum thermalization, Phys. Rev. E 50, 888 (1994).

[4] M. Srednicki, The approach to thermal equilibrium in quantized chaotic systems, J. Phys. A 32, 1163 (1999).

[5] M. Rigol, V. Dunjko, and M. Olshanii, Thermalization and its mechanism for generic isolated quantum systems, Nature (Lond.) 452, 854 (2008).
[6] B. Sutherland, Beautiful Models: 70 Years of Exactly Solved Quantum Many-body Problems (World Scientific, River Edge, NJ, 2004).

[7] Subir Sachdev, Quantum Phase Transitions (Cambridge University Press, Cambridge, UK, 1999).

[8] M. Basko, I. L. Aleiner, and B. L. Altshuler, Metal-insulator transition in a weakly interacting many-electron system with localized single-particle states, Ann. Phys. 321, 1126 (2006).

[9] V. Oganesyan and D. A. Huse, Localization of interacting fermions at high temperature, Phys. Rev. B 75, 155111 (2007).

[10] A. Pal and D. A. Huse, Many-body localization phase transition, Phys. Rev. B 82, 174411 (2010). 
[11] D. A. Huse, R. Nandkishore, V. Oganesyan, A. Pal, and S. L. Sondhi, Localization-protected quantum order, Phys. Rev. B 88, 014206 (2013).

[12] M. Serbyn, Z. Papic, and D. A. Abanin, Local Conservation Laws and the Structure of the Many-Body Localized States, Phys. Rev. Lett. 111, 127201 (2013).

[13] H. Bernien, S. Schwartz, A. Keesling, H. Levine, A. Omran, H. Pichler, S. Choi, A. S. Zibrov, M. Endres, M. Greiner, V. Vuletic, and M. D. Lukin, Probing many-body dynamics on a 51-atom quantum simulator, Nature (Lond.) 551, 579 (2017).

[14] C. J. Turner, A. A. Michailidis, D. A. Abanin, M. Serbyn, and Z. Papic, Weak ergodicity breaking from quantum many-body scars, Nat. Phys. 14, 745 (2018).

[15] C. J. Turner, A. A. Michailidis, D. A. Abanin, M. Serbyn, and Z. Papic, Quantum scarred eigenstates in a Rydberg atom chain: Entanglement, breakdown of thermalization, and stability to perturbations, Phys. Rev. B 98, 155134 (2018).

[16] C.-J. Lin and Olexei I. Motrunich, Exact Quantum Many-Body Scar States in the Rydberg-Blockaded Atom Chain, Phys. Rev. Lett. 122, 173401 (2019).

[17] N. Shiraishi, Connection between quantum-many-body scars and the Affleck-Kennedy-Lieb-Tasaki model from the viewpoint of embedded Hamiltonians, J. Stat. Mech. (2019) 083103.

[18] V. Khemani, C. R. Laumann, and A. Chandran, Signatures of integrability in the dynamics of Rydberg-blockaded chains, Phys. Rev. B 99, 161101(R) (2019).

[19] S. Choi, C. J. Turner, H. Pichler, W. W. Ho, A. A. Michailidis, Z. Papić, M. Serbyn, M. D. Lukin, and D. A. Abanin, Emergent SU(2) Dynamics and Perfect Quantum Many-Body Scars, Phys. Rev. Lett. 122, 220603 (2019).

[20] N. Shiraishi and T. Mori, Systematic Construction of Counterexamples to the Eigenstate Thermalization Hypothesis, Phys. Rev. Lett. 119, 030601 (2017)

[21] M. Schecter and T. Iadecola, Weak Ergodicity Breaking and Quantum Many-Body Scars in Spin-1 XY Magnets, Phys. Rev. Lett. 123, 147201 (2019).

[22] S. Moudgalya, S. Rachel, B. A. Bernevig, and N. Regnault, Exact excited states of nonintegrable models, Phys. Rev. B 98, 235155 (2018).

[23] S. Maudgalya, N. Regnault, and B. A. Bernevig, Entanglement of exact excited states of Affleck-Kennedy-Lieb-Tasaki models: Exact results, many-body scars, and violation of the strong eigenstate thermalization hypothesis, Phys. Rev. B 98, 235156 (2018)

[24] D. K. Mark and O. I. Motrunich, $\eta$-pairing states as true scars in an extended Hubbard model, Phys. Rev. B 102, 075132 (2020).

[25] S. Moudgalya, N. Regnault, and B. A. Bernevig, $\eta$-pairing in Hubbard models: From spectrum generating algebras to quantum many-body scars, Phys. Rev. B 102, 085140 (2020).

[26] W. W. Ho, S. Choi, H. Pichler, and M. D. Lukin, Periodic Orbits, Entanglement, and Quantum Many-Body Scars in Constrained Models: Matrix Product State Approach, Phys. Rev. Lett. 122, 040603 (2019).

[27] K. Bull, I. Martin, and Z. Papic, Systematic Construction of Scarred Many-Body Dynamics in 1D Lattice Models, Phys. Rev. Lett. 123, 030601 (2019).

[28] S. Pai and M. Pretko, Dynamical Scar States in Driven Fracton Systems, Phys. Rev. Lett. 123, 136401 (2019).
[29] B. Mukherjee, S. Nandy, A. Sen, D. Sen, and K. Sengupta, Collapse and revival of quantum many-body scars via Floquet engineering, Phys. Rev. B 101, 245107 (2020).

[30] B. Mukherjee, A. Sen, D. Sen, and K. Sengupta, Restoring coherence via aperiodic drives in a many-body quantum system, Phys. Rev. B 102, 014301 (2020).

[31] B. Mukherjee, A. Sen, D. Sen, and K. Sengupta, Dynamics of the vacuum state in a periodically driven Rydberg chain, Phys. Rev. B 102, 075123 (2020).

[32] S. Sugiura, T. Kuwahara, and K. Saito, Many-body scar state intrinsic to periodically driven system, Phys. Rev. Res. 3, L012010 (2021).

[33] K. Mizuta, K. Takasan, and Norio Kawakami, Exact Floquet quantum many-body scars under Rydberg blockade, Phys. Rev. Res. 2, 033284 (2020).

[34] N. Shibata, N. Yoshioka, and H. Katsura, Onsager's Scars in Disordered Spin Chains, Phys. Rev. Lett. 124, 180604 (2020).

[35] S. Maudgalya, N. Regnault, and B. A. Bernevig, Quantum many-body scars in a Landau level on a thin torus, Phys. Rev. B 102, 195150 (2020).

[36] S. Ok, K. Choo, C. Mudry, C. Castelnovo, C. Chamon, and T. Neupert, Topological many-body scar states in dimensions one, two, and three, Phys. Rev. Res. 1, 033144 (2019).

[37] C.-J. Lin, V. Calvera, and T. H. Hsieh, Quantum many-body scar states in two-dimensional Rydberg atom arrays, Phys. Rev. B 101, 220304(R) (2020).

[38] A. A. Michailidis, C. J. Turner, Z. Papić, D. A. Abanin, and M. Serbyn, Stabilizing two-dimensional quantum scars by deformation and synchronization, Phys. Rev. Res. 2, 022065(R) (2020).

[39] K. Lee, R. Melendrez, A. Pal, and H. J. Changlani, Exact threecolored quantum scars from geometric frustration, Phys. Rev. B 101, 241111(R) (2020).

[40] N. S. Srivatsa, J. Wildeboer, A. Seidel, and A. E. B. Nielsen, Quantum many-body scars with chiral topological order in two dimensions and critical properties in one dimension, Phys. Rev. B 102, 235106 (2020).

[41] J. Wildeboer, A. Seidel, N. S. Srivatsa, A. E. B. Nielsen, and O. Erten, Topological quantum many-body scars in quantum dimer models on the Kagome lattice, arXiv:2009.00022.

[42] A. J. A. James, R. M. Konik, and N. J. Robinson, Symmetry Breaking Bias and the Dynamics of a Quantum Phase Transition, Phys. Rev. Lett. 122, 130603 (2019).

[43] F. M. Surace, P. P. Mazza, G. Giudici, A. Lerose, A. Gambassi, and M. Dalmonte, Lattice Gauge Theories and String Dynamics in Rydberg Atom Quantum Simulators, Phys. Rev. X 10, 021041 (2020).

[44] G. Magnifico, M. Dalmonte, P. Facchi, S. Pascazio, F. V Pepe, and E. Ercolessi, Real time dynamics and confinement in the $\mathbb{Z}_{n}$ Schwinger-Weyl lattice model for 1+1 QED, Quantum 4, 281 (2020).

[45] B. Mukherjee, D. Banerjee, K. Sengupta, and A. Sen, A minimal model for Hilbert space fragmentation with local constraints, arXiv:2106.14897v2.

[46] T. Iadecola, M. Schecter, and S. Xu, Quantum many-body scars from magnon condensation, Phys. Rev. B 100, 184312 (2019).

[47] D. Banerjee and A. Sen, Quantum Scars from Zero Modes in an Abelian Lattice Gauge Theory, Phys. Rev. Lett. 126, 220601 (2021). 
[48] V. Karle, M. Serbyn, and A. A. Michailidis, Area-Law Entangled Eigenstates from Nullspaces of Local Hamiltonians, Phys. Rev. Lett. 127, 060602 (2021).

[49] V. Khemani, M. Hermele, and R. M. Nandkishore, Localization from Hilbert space shattering: From theory to physical realizations, Phys. Rev. B 101, 174204 (2020).

[50] P. Sala, T. Rakovszky, R. Verresen, M. Knap, and F. Pollmann, Ergodicity Breaking Arising from Hilbert Space Fragmentation in Dipole-Conserving Hamiltonians, Phys. Rev. X 10, 011047 (2020).

[51] T. Iadecola and M. Schecter, Quantum many-body scar states with emergent kinetic constraints and finite-entanglement revivals, Phys. Rev. B 101, 024306 (2020).

[52] Z. C. Yang, F. Liu, A. V. Gorshkov, and T. Iadecola, HilbertSpace Fragmentation from Strict Confinement, Phys. Rev. Lett. 124, 207602 (2020).

[53] K. Lee, A. Pal, and H. J. Changlani, Frustration-induced emergent Hilbert space fragmentation, Phys. Rev. B 103, 235133 (2021).
[54] D. Hahn, P. A. McClarty, and D. J. Luitz, Information dynamics in a model with Hilbert space fragmentation, arxiv:2104.00692v4.

[55] S. Roy and A. Lazarides, Strong ergodicity breaking due to local constraints in a quantum system, Phys. Rev. Res. 2, 023159 (2020).

[56] S. Scherg, T. Kohlert, P. Sala, F. Pollmann, B. H. Madhusudhana, I. Bloch, and M. Aidelsburger, Observing non-ergodicity due to kinetic constraints in tilted Fermi-Hubbard chains, Nat. Commun. 12, 4490 (2021).

[57] A. Signoles, T. Franz, R. Ferracini Alves, M. Gärttner, S. Whitlock, G. Zü, and M. Weidemüller, Observation of Glassy Dynamics in a Disordered Quantum Spin System, Phys. Rev. X 11, 011011 (2021).

[58] B. Nienhuis, M. Campostrini, and P. Calabrese, Entanglement, combinatorics and finite-size effects in spin-chains, J. Stat. Mech. (2009) P02063. 